\title{
РАСЧЕТ ТЕМПЕРАТУРНОГО РЕЖИМА МЕРЗЛОГО ГРУНТА ВОКРУГ ТРУБОПРОВОДОВ \\ (программный продукт для ЭВМ)
}

\section{Моисеев Б.В., Гербер А.Д., Илюхин К.Н., Налобин Н.В.}

Тюменский государственный архитектурно-строительный университет, Тюмень, Россия (625001, Тюмень, ул. Луначарского, 2), e-mail: Mr-Fahrenheit@yandex.ru

Обустройство нефтяных и газовых месторождений требует сооружения различных трубопроводов (водопроводов, газопроводов и нефтепроводов) подземной прокладкой. При эксплуатации трубопроводов в мерзлых грунтах происходит теплообмен между ними и образование талой зоны, что приводит к деформации трубопроводов. Условия строительства и эксплуатации трубопроводов существенно связаны с их температурным режимом. В связи с этим необходимо знать закономерности формирования ореолов протаивания вокруг трубопроводов. Один из способов ограничения теплового воздействия трубопровода на мерзлый грунт - применение теплоизоляции.

Для выбора оптимальной толщины изоляции трубопроводов необходимо иметь методику расчета. На параметры режима работы теплопроводов и газопроводов большое влияние оказывает температурное поле грунта вокруг трубопроводов. Таким образом, выяснение закономерностей формирования ореолов протаивания вокруг трубопроводов и определение оптимальных условий их прокладки при строительстве в районах с вечномерзлыми грунтами на севере Тюменской области является весьма актуальной задачей. Для построения температурного поля мерзлого грунта авторами был разработан алгоритм и программа на ЭВМ. Было выполнено решение некоторых задач на основе полученных зависимостей и построены графики зависимости. Исследования и выполненные расчеты по подземной прокладке трубопроводов позволили авторам дать рекомендации, направленные на увеличение надежности трубопроводов.

Ключевые слова: теплопровод, тепловая изоляция, мерзлые грунты, распределение температуры 


\section{TEMPERATURE CONDITION CALCULATION \\ OF FROST SOIL AROUND PIPELINES \\ (program product for computer)}

\section{Moiseev B.V., Gerber A.D., Ilyukhin K.N., Nalobin N.V.}

Tyumen State Architectural and Building University (Lunacharskogo street, 2, 625001, Tyumen, Russia), e-mail: Mr-Fahrenheit@yandex.ru

Development of the infrastructure of oil and gas fields require construction of different pipelines (water, gas and oil pipelines) by means of underground laying. When using pipelines in frozen soils, heat exchange between them and formation of a melted area occur that causes pipeline deformation. The building and operation conditions of pipelines are to a considerable extent connected with their temperature conditions. Due to this it is necessary to know the regularities of formation of the thawing haloes around the pipelines. One of the methods of limitation of the pipeline thermal effect on the frozen soil is use of heat insulation.

In order to choose an optimal pipeline insulation thickness it is necessary to have a calculation method. A great influence on heat and gas pipeline operating mode parameters is exerted by the soil thermal field around the pipelines. Therefore, determination of the regularities of formation of the thawing haloes around the pipelines and determination of the optical conditions of their laying for construction in the areas permanently frozen soils in the north of the Tyumen Region is quite a vital task. In order to determine the frozen soil thermal field the authors developed an algorithm and the program on computer. Some tasks were solved and dependence diagrams were constructed based on the obtained dependences. The studies and the made calculations one the underground pipeline laying allowed to the authors to give recommendations aimed at the pipeline reliability growth.

Keywords: heat pipeline, heat insulation, permafrost soils, temperature distribution 


\section{Введение}

Для снижения трудоемкости управления системами инженерных коммуникаций необходимо на стадии проектирования рассматривать различные модели оптимизации работы этих систем, что достигается путем использования компьютерных информационных технологий. С этой целью авторами был разработан алгоритм и компьютерная программа. Численный эксперимент выгодно отличается от лабораторного или промышленного тем, что есть возможность исследовать влияние каждого определяющего параметра изучаемого явления в отдельности в широком диапазоне значений.

Программа для ЭВМ «Расчет температурного режима мерзлого грунта вокруг трубопроводов», разработанная авторами в 2016 г., позволяет моделировать температурный режим мерзлого грунта вокруг трубопроводов и производить основные расчеты, выполняемые при проектировании и эксплуатации трубопроводных систем. С помощью программы можно рассчитать: зависимость температуры грунта от времени в месте заложения трубопровода; зависимость температуры грунта от глубины заложения трубопровода; зависимость температуры на поверхности трубы (поверхность изоляции) от глубины заложения трубопровода в выбранном сечении; зависимость величины потока тепла с поверхности трубы от глубины заложения трубопровода в выбранном сечении; зависимость температуры жидкости в теплопроводе при заданном расходе, толщине изоляции от расстояния до входа (падение температуры вдоль теплопровода); зависимость значения толщины изоляционного слоя при заданном перепаде температур от расстояния до входа; зависимость размеров зоны оттаивания (верхняя и нижняя граница) от глубины заложения трубопровода при заданных значениях перепада температур и толщине изоляционного слоя.

Все полученные графики зависимостей могут быть сохранены в виде рисунков в формате точечного рисунка. Для выполнения расчетов необходимо выбрать нужный вид расчета (опция меню «Расчет» на панели инструментов или воспользоваться пиктографической кнопкой). Рассчитанные графики выводятся на экран и печать. Использование программы позволяет выбрать наиболее оптимальные решения, что приведет к снижению себестоимости теплоты и затрат топлива.

Программа может применяться в проектных, эксплуатационных организациях, диспетчерских и управляющих службах, а также в вузах, как составляющие системы информатизации образования. 


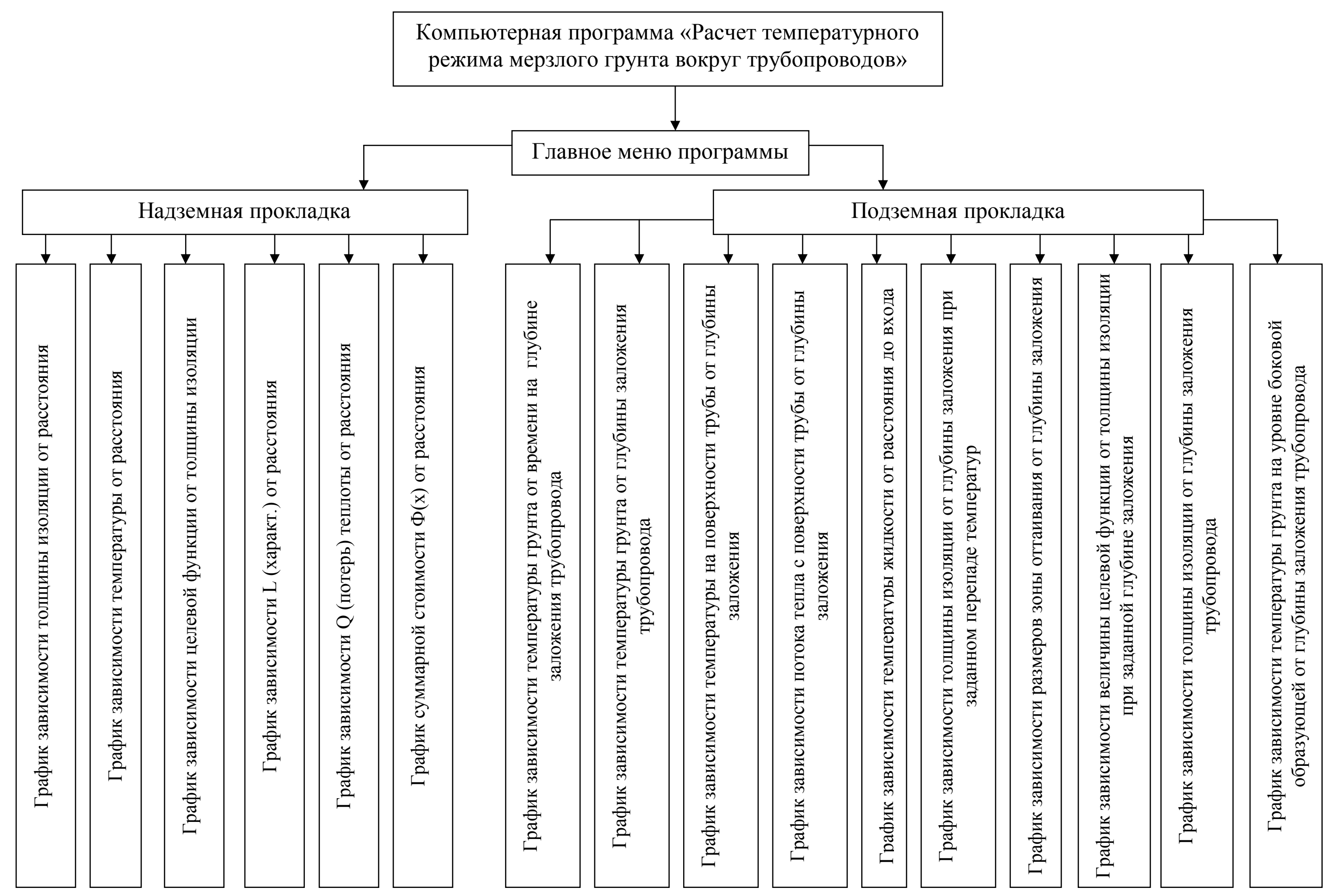




\section{1 Общее описание программы}

Программа написана в стиле многодокументного приложения (MDI).

В главном окне программы задаются основные исходные данные, производится вызов диалоговых окон расчетов, печать, просмотр ранее выполненных расчетов. Главное окно программы показано на рис. 1.

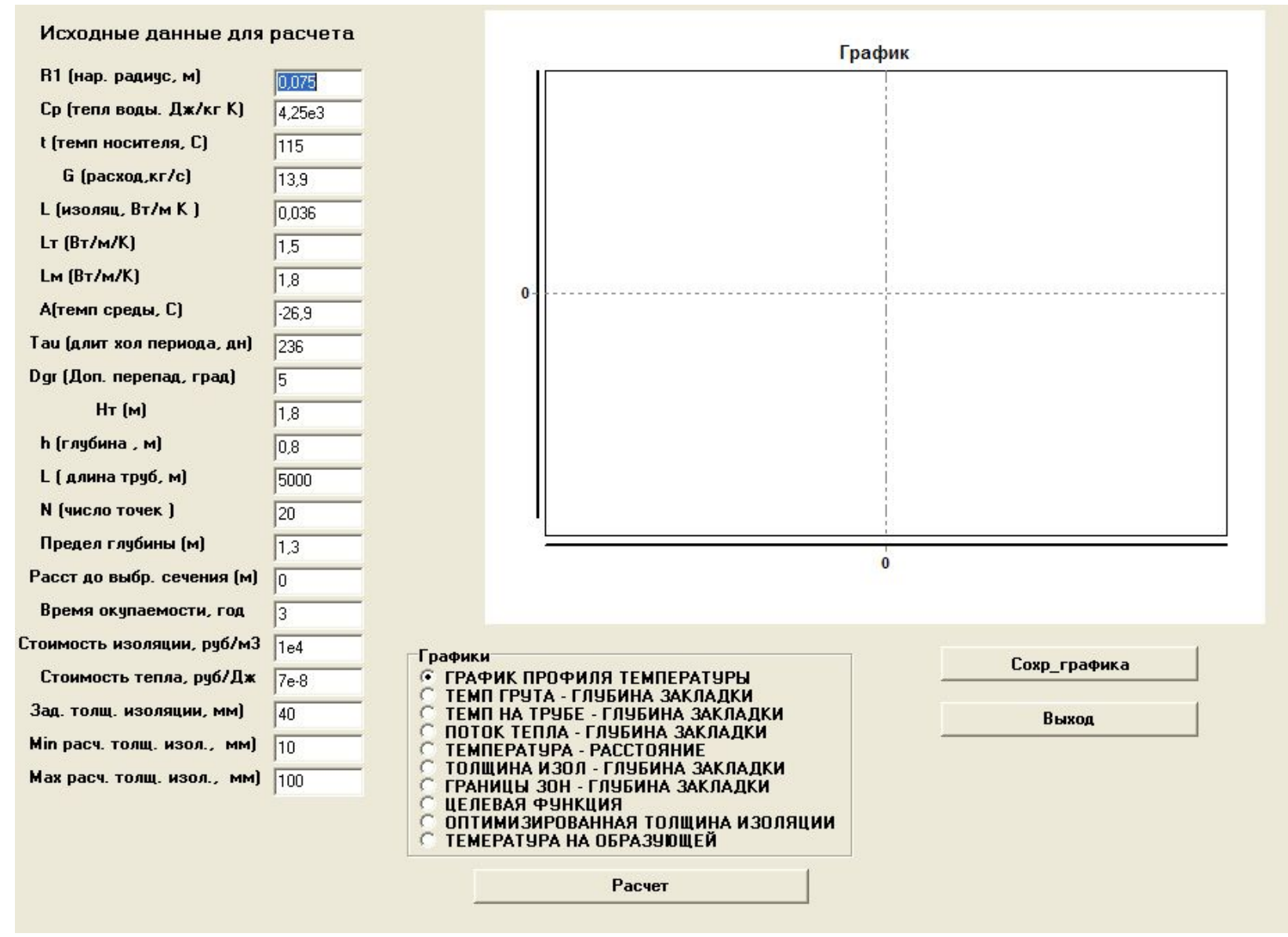

Рисунок 1 - Главное окно программы

В программу входит комплекс расчетов:

1. Определение температуры грунта от времени на глубине заложения трубопровода.

2. Построение графика зависимости температуры грунта от глубины заложения трубопровода.

3. Определение зависимости температуры на поверхности трубы от глубины заложения трубопровода.

4. Определение зависимости потока теплоты с поверхности трубы от глубины заложения трубопровода.

5. Определение зависимости температуры теплоносителя от расстояния. 
6. Определение зависимости толщины изоляции от глубины заложения при заданном перепаде температур.

7. Определение зависимости толщины изоляции от расстояния.

8. Определение зависимости размеров оттаивания от глубины заложения трубопровода.

9. Определение зависимости величины целевой функции от толщины изоляции при заданной глубине заложения трубопровода.

10. Определение зависимости толщины изоляции от глубины заложения трубопровода.

11. Определение зависимости температуры грунта на уровне боковой образующей от глубины заложения трубопровода.

2. Математическая модель температурного режима в ППУ-изоляции теплопровода при подземной бесканальной прокладке в вечномерзлых грунтах

Авторами проведен анализ существующих методов тепловых расчетов трубопроводов жизнеобеспечения. Из анализа нормативных и расчетных потерь теплоты теплопроводами выявлены значительные расхождения. Задачей теплового расчета трубопроводов является определение температурного режима грунта вокруг трубопровода. Это важно для определения толщины изоляционного слоя, предназначенного для уменьшения потерь теплоты в окружающую среду и исключения теплового воздействия на мерзлый грунт. Одним из способов ограничения теплового воздействия трубопровода на мерзлый грунт применение теплоизоляции (ППУ - изоляция с коэффициентом теплопроводности $\left.\lambda=0,036 \mathrm{Bт} /\left(\mathrm{M}^{\circ}{ }^{\circ} \mathrm{C}\right)\right)$. Рассмотрим теплопровод, проложенный в вечномерзлом грунте с температурой грунта $\mathrm{t}_{\text {гр }}=-2^{\circ} \mathrm{C}$ на глубине прокладки теплопровода для г. Н.-Уренгой.

Для постановки и решения задачи были приняты следующие допущения: взаимное влияние температурных полей прямого и обратного трубопроводов тепловых сетей при двухтрубной бесканальной прокладке отсутствует; средняя глубина заложения теплопровода в вечномерзлом грунте от 1 до 1,5 м; грунт был представлен кольцеобразной мерзлой зоной вокруг теплопровода без учета влияния наружного воздуха на грунт. С учетом принятых условий расчетная модель процесса теплового режима вокруг подземного теплопровода, представлена на рис. 2. 


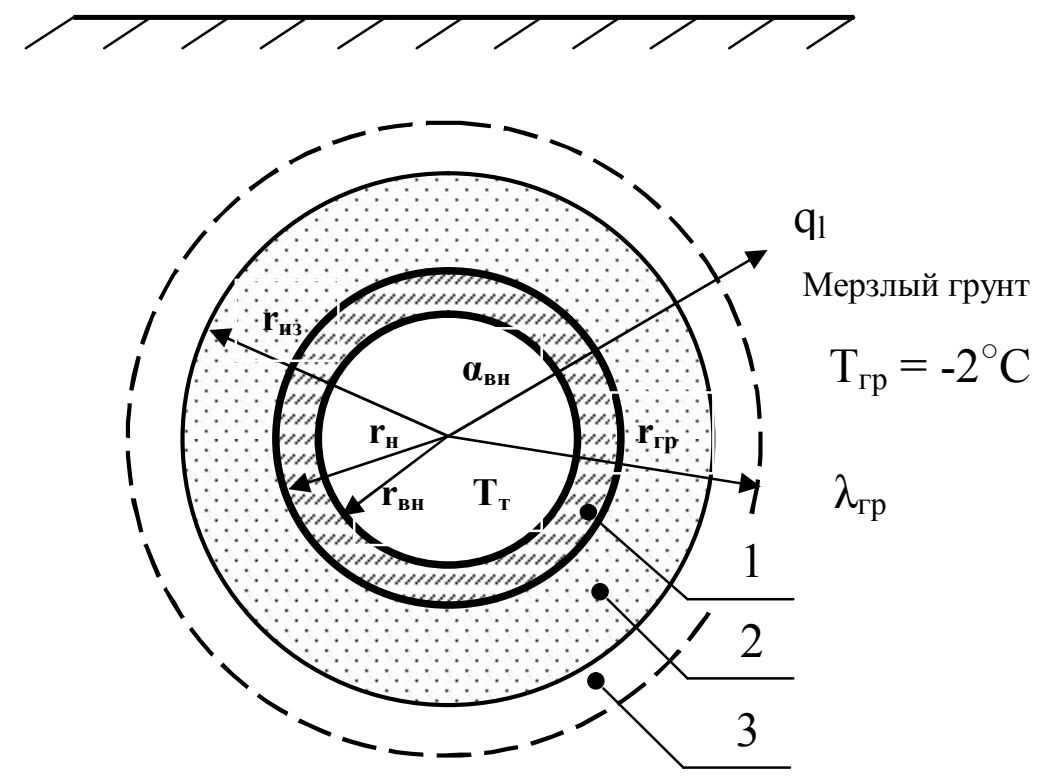

Рисунок 2 - Расчетная модель формирования температурного режима теплопровода в вечномерзлых грунтах

Распределение температур в слое изоляции трубопровода описывается дифференциальным уравнением в частных производных, которое в цилиндрических координатах имеет вид:

$$
\begin{aligned}
& \frac{\partial \mathrm{t}_{\mathrm{i}}}{\partial \tau}=\mathrm{a}_{\mathrm{i}}\left(\frac{\partial^{2} \mathrm{t}_{\mathrm{i}}}{\partial \mathrm{r}^{2}}+\frac{1}{\mathrm{r}} \frac{\partial \mathrm{t}_{\mathrm{i}}}{\partial \mathrm{r}}\right), \\
& \mathrm{r}_{\mathrm{i}-1} \leq \mathrm{r} \leq \mathrm{r}_{\mathrm{i}} ; \quad \mathrm{i}=1,2, \ldots, \mathrm{N} .
\end{aligned}
$$

- на внутренней поверхности теплопровода

$$
\lambda_{1}\left(\frac{\partial \mathrm{t}_{1}}{\partial \mathrm{r}}\right)_{\mathrm{B}}=-\alpha_{1}\left(\mathrm{t}_{\mathrm{T}}-\mathrm{t}_{1}\right), \mathrm{r}=\mathrm{r}_{\mathrm{BH}}
$$

- на стыках различных слоев изоляции

$$
\lambda_{i} \frac{\partial t_{i}}{\partial r_{i}}=\lambda_{i+1} \frac{\partial t_{i+1}}{\partial r_{i+1}}, r=r_{i}(i=1,2, \ldots, N-1) ;
$$

- на стыке наружной поверхности изоляции с грунтом

$$
\lambda_{\mathrm{N}} \frac{\partial \mathrm{t}_{\mathrm{N}}}{\partial \mathrm{r}_{\mathrm{N}}}=\lambda_{\mathrm{rp}} \frac{\partial \mathrm{t}_{\mathrm{rp}}}{\partial \mathrm{r}_{\mathrm{N}+1}}, \mathrm{r}=\mathrm{r}_{\mathrm{N}} ; \mathrm{t}_{\mathrm{rp}}=\mathrm{t}_{\mathrm{N}}, \mathrm{r}=\mathrm{r}_{\mathrm{N}+1}
$$

где $\alpha_{1}-$ коэффициент теплоотдачи от теплоносителя к стенке теплопроводами, $\mathrm{BT} /\left(\mathrm{M}^{2} \cdot{ }^{\circ} \mathrm{C}\right) ; \mathrm{r}_{\text {вн }}$ - внутренний радиус теплопровода, м; $\lambda_{\text {гр }}-$ коэф-т теплопроводности грунта, Вт/(м $\left.\cdot{ }^{\circ} \mathrm{C}\right) ; \alpha_{\mathrm{i}}$ - температуропроводность слоя теплоизоляции, $\mathrm{m}^{2} / \mathrm{c} ; \mathrm{t}$ - температура, ${ }^{\circ} \mathrm{C}$; $\tau$ - время, с; $r_{i}$ - текущее значение радиуса трубы, м. 
Теплофизические свойства грунта и теплоизоляции определяются по среднеинтегральной температуре. Грунт был представлен кольцеобразной мерзлой зоной вокруг теплопровода без учета влияния дневной поверхности, так как на глубине заложения теплопровода мерзлый грунт по данным изыскания проектной организации ОАО «Тюменьгеолпроект» имеет круглогодичную температуру грунта $\mathrm{t}_{\text {гр }}=-2{ }^{\circ} \mathrm{C}$ для г. Новый Уренгой.

\section{3. Описание алгоритма построения температурного поля мерзлого грунта}

Описание возможностей программы для расчета теплового режима теплопровода в варианте его подземной бесканальной прокладки.

На форме присутствует несколько окон, определяющих значения геометрических характеристик теплопровода, а также теплофизические характеристики грунта и изоляции. В частности:

$R 1$ - внешний радиус трубы без изоляции,

$C_{\mathrm{p}}$ - теплоемкость теплоносителя (обычно воды),

$t$ - температура воды на входе,

$G$ - массовый расход воды,

$L$ - коэффициент теплопроводности изоляции,

$L_{\mathrm{T}}$ - коэффициент теплопроводности талого грунта,

$L_{\mathrm{m}}$ - коэффициент теплопроводности мерзлого грунта,

$A$ - максимальное значение амплитуды температуры холодного периода,

$\tau_{\text {х }}$ - длительность холодного периода,

$\Delta t_{0}$ - допустимый перепад температур, задаваемый изначально,

$H_{\text {т }}$ - глубина сезонного промерзания грунта, определяется по картам изолиний,

$h$ - глубина заложения трубопровода,

$H_{\text {пр }}$ - предельная глубина закладки трубопровода, используется при расчете размеров зон оттаивания.

Для построения температурного поля мерзлого грунта авторами был разработан алгоритм. Сезонное изменение температуры на поверхности грунта было представлено в следующем виде $\mathrm{t}_{\mathrm{H}}(\tau)=\mathrm{A} \sin \left(\frac{\pi \tau}{\tau_{\mathrm{x}}}\right)$, где $\tau$ - время отсчета с момента наступления холодного периода, в днях или месяцах, $\tau_{\text {x }}$ - продолжительность холодного периода сезонного изменения температуры $\left(\tau_{\mathrm{x}}=236\right.$ суток или $\tau_{\mathrm{x}}=7.76$ месяца для района Н.Уренгой, A =-26.9 градусов - амплитуда колебания температуры наружного воздуха). 
Глубина сезонного промерзания или уровень ВМГ (вечномерзлых грунтов) $\mathrm{H}_{\mathrm{T}}=2 \mathrm{M}$ для песчаных грунтов или $\mathrm{H}_{\mathrm{T}}=1.7 \mathrm{M}$ для глинистых грунтов. Зависимость глубины промерзания от времени $\mathrm{H}_{\mathrm{m}}(\tau)=\mathrm{H}_{\mathrm{T}} \sqrt{\frac{\tau}{\tau_{\mathrm{x}}}}$.

Зависимость температуры на глубине заложения трубопровода тогда можно представить в следующей форме $\mathrm{t}_{\mathrm{h}}=\mathrm{t}_{\mathrm{H}}(\tau)\left(1-\frac{\mathrm{h}}{\mathrm{H}_{\mathrm{m}}(\tau)}\right)$. После подстановки $\mathrm{t}_{\mathrm{H}}(\tau)$ в это выражение получим:

$$
\mathrm{t}_{\mathrm{h}}=\mathrm{A} \sin \left(\frac{\pi \tau}{\tau_{\mathrm{x}}}\right)\left(1-\frac{\mathrm{h}}{\mathrm{H}_{\mathrm{M}}(\tau)}\right) .
$$

Кроме того, время когда фронт температуры будет на глубине заложения определяется из $\tau_{0}=\tau_{\mathrm{x}}\left(\frac{\mathrm{h}}{\mathrm{H}_{\mathrm{T}}}\right)^{2}$.

Таким образом, изменения температуры на глубине заложения трубопровода $\mathrm{h}$, можно рассчитать по следующей зависимости. Наиболее холодная температура на этой глубине равна минимуму данной функции, и именно она будет участвовать в дальнейших расчетах.

$$
\mathrm{t}_{\mathrm{h}}=\left\{\begin{array}{c}
0 ; \tau<\tau_{0} \\
\mathrm{~A} \sin \left(\frac{\pi \tau}{\tau_{\mathrm{x}}}\right)\left(1-\frac{\mathrm{h}}{\mathrm{H}_{\mathrm{M}}(\tau)}\right) ; \tau_{0} \leq \tau \leq \tau_{\mathrm{x}} . \\
0 ; \tau<\tau_{0}
\end{array}\right.
$$

На следующем этапе найдем толщину изоляции обеспечивающей приемлемое качество доставки теплоносителя, которое определяется условием заданного уровня падения температуры теплоносителя по длине теплопровода $\mathrm{t}^{\prime}-\mathrm{t}^{\prime \prime}<\Delta \mathrm{t}_{0}, \mathrm{t}^{\prime}$ температура на входе, $\mathrm{t}^{\prime \prime}$ - температура на конце трубопровода, $\Delta \mathrm{t}_{0} \approx 7^{\circ} \mathrm{C}$ - допустимое падение температуры.

Для определения температуры на поверхности изоляции составим уравнение баланса тепловых потоков.

Поток со стороны горячей воды к поверхности трубы

$$
\mathrm{q}_{1}=\frac{2 \pi \lambda_{\text {из }}\left(\mathrm{t}_{\text {ж }}-\mathrm{t}_{\mathrm{T}}\right)}{\ln \frac{\mathrm{D}_{\mathrm{H}}+2 \delta_{\text {из }}}{\mathrm{D}_{\text {н }}}}=\frac{2 \pi \lambda_{\text {из }}\left(\mathrm{t}_{\text {ж }}-\mathrm{t}_{\mathrm{T}}\right)}{\alpha} .
$$


Поток со стороны горячей трубы к мерзлому грунту

$$
\mathrm{q}_{1}=\frac{2 \pi \lambda_{\text {гр }}\left(\mathrm{t}_{\mathrm{T}}-\mathrm{t}_{\text {гр }}\right)}{\ln \left[\frac{2 \mathrm{~h}}{\mathrm{D}_{\text {н }}+2 \delta_{\text {из }}}+\sqrt{\left(\frac{2 \mathrm{~h}}{\mathrm{D}_{\text {н }}+2 \delta_{\text {из }}}\right)^{2}-1}\right]}=\frac{2 \pi \lambda_{\text {гр }}\left(\mathrm{t}_{\mathrm{T}}-\mathrm{t}_{\mathrm{rp}}\right)}{\beta} .
$$

Уравнение баланса в данном случае можно записать

$$
\frac{\mathrm{q}_{1} \alpha}{2 \pi \lambda_{\text {из }}}+\frac{\mathrm{q}_{1} \beta}{2 \pi \lambda_{\text {гр }}}=\mathrm{t}_{\text {ж }}-\mathrm{t}_{\text {гр }},
$$

где $\lambda_{\text {гр }}-$ усредненная теплопроводность равная среднему значению мерзлого и талого грунта.

Отсюда можно получить значение величины потока

$$
\mathrm{q}_{1}=\frac{\mathrm{t}_{\text {ж }}-\mathrm{t}_{\text {гр }}}{\frac{\alpha}{2 \pi \lambda_{\text {из }}}+\frac{\beta}{2 \pi \lambda_{\text {гр }}}} .
$$

Зная поток можно рассчитать температуру грунта в месте заложения теплопровода.

$$
\mathrm{t}_{\mathrm{T}}=\mathrm{t}_{\text {ж }}-\frac{\alpha \mathrm{q}_{1}}{2 \pi \lambda_{\text {из }}} .
$$

Падение температуры жидкости по длине теплопровода можно найти по следующей формуле

$$
\mathrm{t}_{\text {ж }}(\mathrm{x})=\mathrm{t}_{\mathrm{rp}}+\left(\mathrm{t}^{\prime}-\mathrm{t}_{\mathrm{rp}}\right) \exp \left(-\frac{\mathrm{x}}{1_{\text {хар }}}\right),
$$

где $\quad 1_{\text {хар }}=\mathrm{GC}_{\text {ж }} \mathrm{R}=\mathrm{GC}_{\text {ж }}\left(\frac{\alpha}{2 \pi \lambda_{\text {из }}}+\frac{\beta}{2 \pi \lambda_{\text {гр }}}\right)$.

Температура на конечном пункте потребления

$$
\mathrm{t}^{\prime \prime}(\mathrm{x})=\mathrm{t}_{\text {ж}}^{\prime \prime}(\mathrm{x})=\mathrm{t}_{\text {гр }}+\left(\mathrm{t}^{\prime}-\mathrm{t}_{\text {гр }}\right) \exp \left(-\frac{\mathrm{L}}{1_{\text {хар }}}\right)
$$

где $\quad \mathrm{L}$ - общая длина трубопровода.

Было выполнено решение некоторых задач сформулированных на основе перечисленных зависимостей.

\section{Определение температуры грунта в месте заложения подземного теплопровода}

Для построения графика зависимости температуры грунта от времени на глубине заложения трубопровода обратимся к диалоговому окну расчета (рис. 3), где, задав исходные данные и, выбрав «график профиля температуры», нажмем кнопку «расчет», получив соответствующий график. 
Диалоговое окно на рис. 4 позволит построить график зависимости температуры грунта от глубины заложения трубопровода.
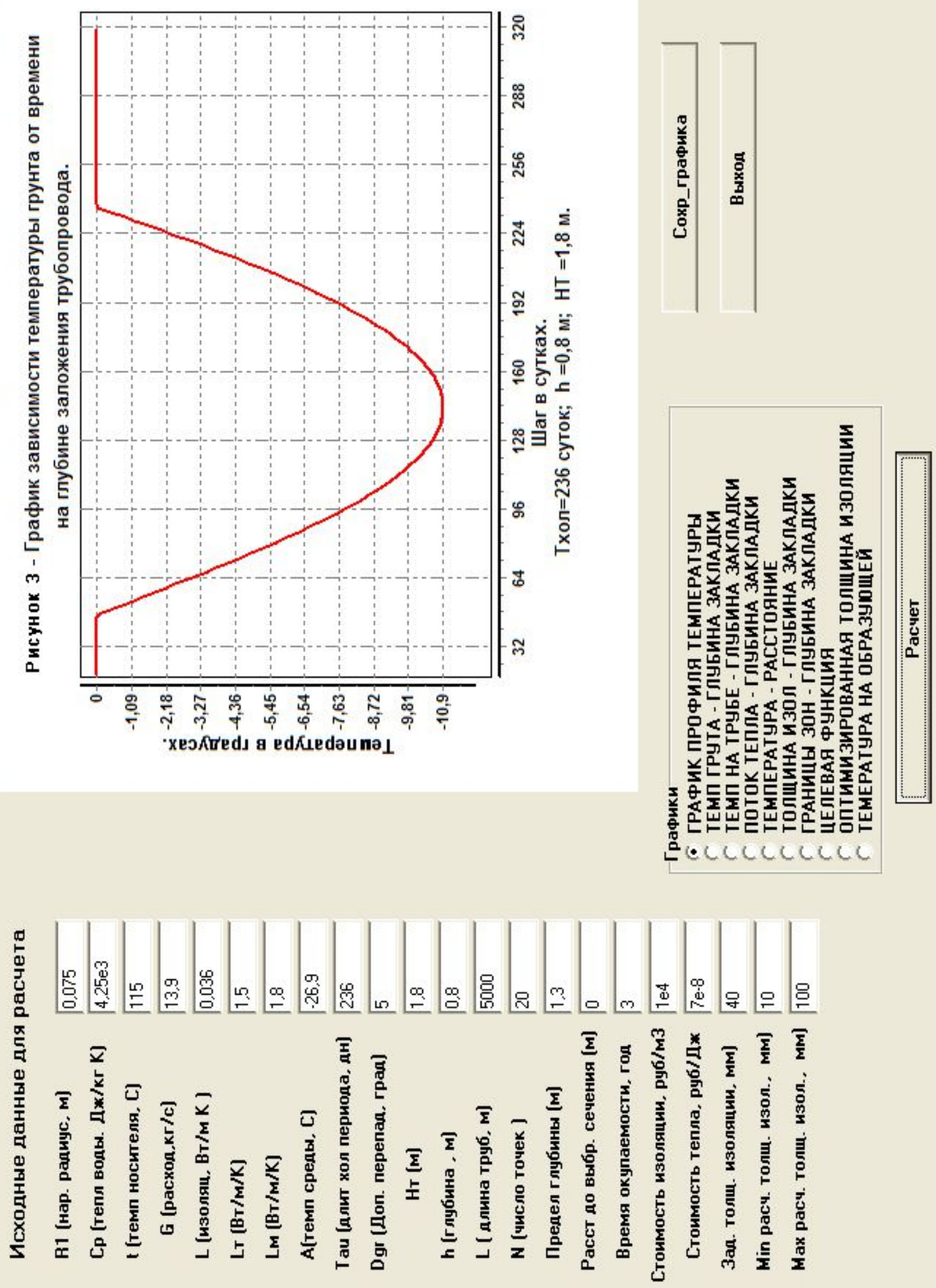

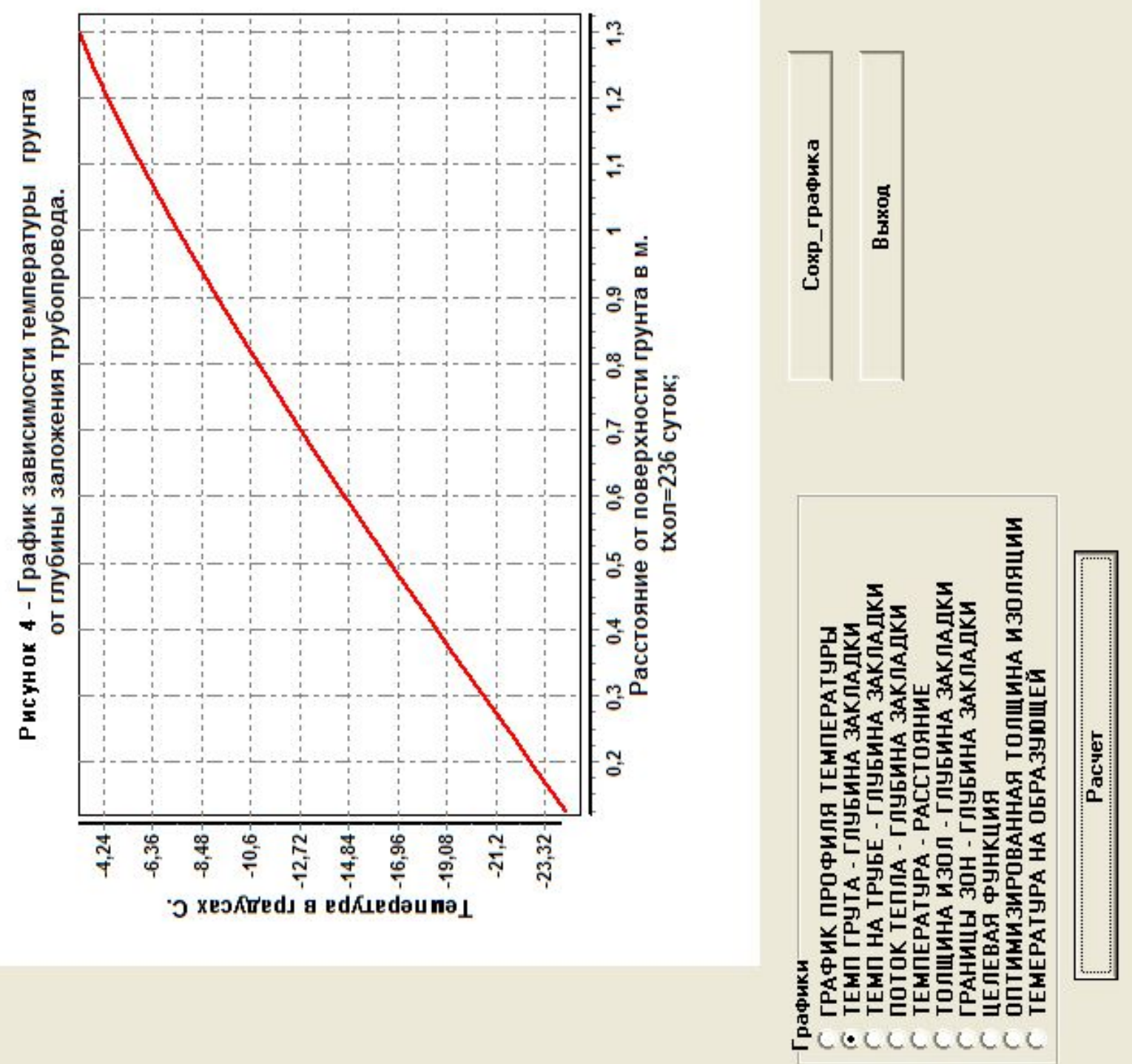

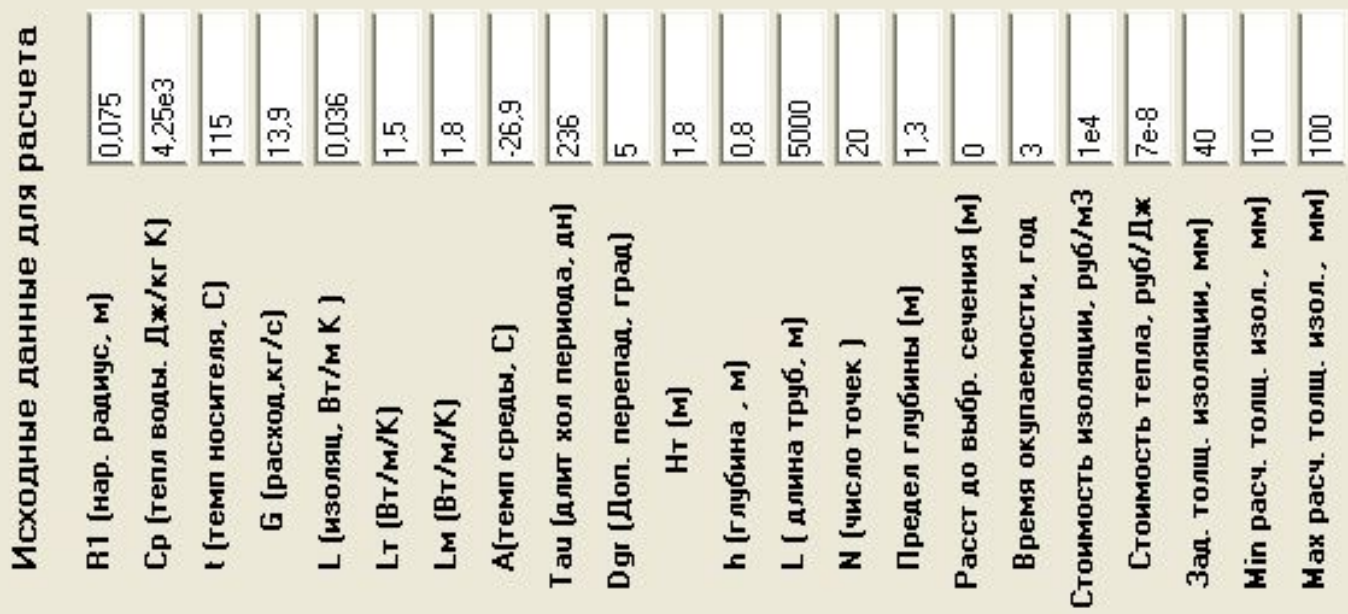




\section{Определение температуры на поверхности трубы и теплового потока при из-} вестной толщине изоляции

В зависимости от толщины изоляции $\delta_{\text {из }}$, которую задаем, например, по СНиП, используя известные значения $\mathrm{t}_{\text {ж }}$ и $\mathrm{t}_{\text {гр }}$, определяем из уравнения баланса потоков теплоты, поток от теплой трубы направленный в грунт и температуру на поверхности изоляции теплопровода. Для этого найдем $\alpha, \beta$, определим суммарное сопротивление $\mathrm{R}$ и

$$
\begin{aligned}
\mathrm{q}_{1} & =\frac{\mathrm{t}_{\text {ж }}-\mathrm{t}_{\mathrm{rp}}}{\mathrm{R}}, \\
\text { где } \quad \mathrm{R} & =\frac{\alpha}{2 \pi \lambda_{\text {из }}}+\frac{\beta}{2 \pi \lambda_{\text {гр }}}, \\
\alpha & =\ln \frac{\mathrm{D}_{\mathrm{H}}+2 \delta_{\text {из }}}{\mathrm{D}_{\mathrm{H}}} ; \\
\beta & =\ln \left(\frac{2 \mathrm{~h}}{\mathrm{D}_{\mathrm{H}}+2 \delta_{\text {из }}}+\sqrt{\left.\left(\frac{2 \mathrm{~h}}{\mathrm{D}_{\mathrm{H}}+2 \delta_{\text {из }}}\right)^{2}-1\right)}-\right.\text { определяются геометрическими размерами }
\end{aligned}
$$

трубы и изоляционного слоя.

Диалоговое окно на рис. 5 дает зависимость температуры на поверхности трубы от глубины заложения трубопровода. Диалоговое окно на рис. 6 позволяет определить зависимость потока теплоты с поверхности трубы от глубины заложения.

Зависимость температуры жидкости в трубопроводе от расстояния при заданных параметрах конструкции определяется по формуле

$$
\mathrm{t}_{\text {ж }}(\mathrm{x})=\mathrm{t}_{\mathrm{rp}}+\left(\mathrm{t}^{\prime}-\mathrm{t}_{\mathrm{rp}}\right) \exp \left(-\frac{\mathrm{x}}{1_{\text {хар }}}\right) .
$$

Диалоговое окно на рис. 7 позволяет определить зависимость температуры жидкости от расстояния.

Определение толщины изоляции в зависимости от глубины заложения при заданном уровне тепловых потерь (температурном перепаде)

Пусть, при известной длине трубопровода L, задан конечный перепад температур $\Delta \mathrm{t}_{0}$ или допустимое падение температуры теплоносителя, или, как возможный вариант, температура в конечном пункте потребления $\mathrm{t}^{\prime \prime}$. 

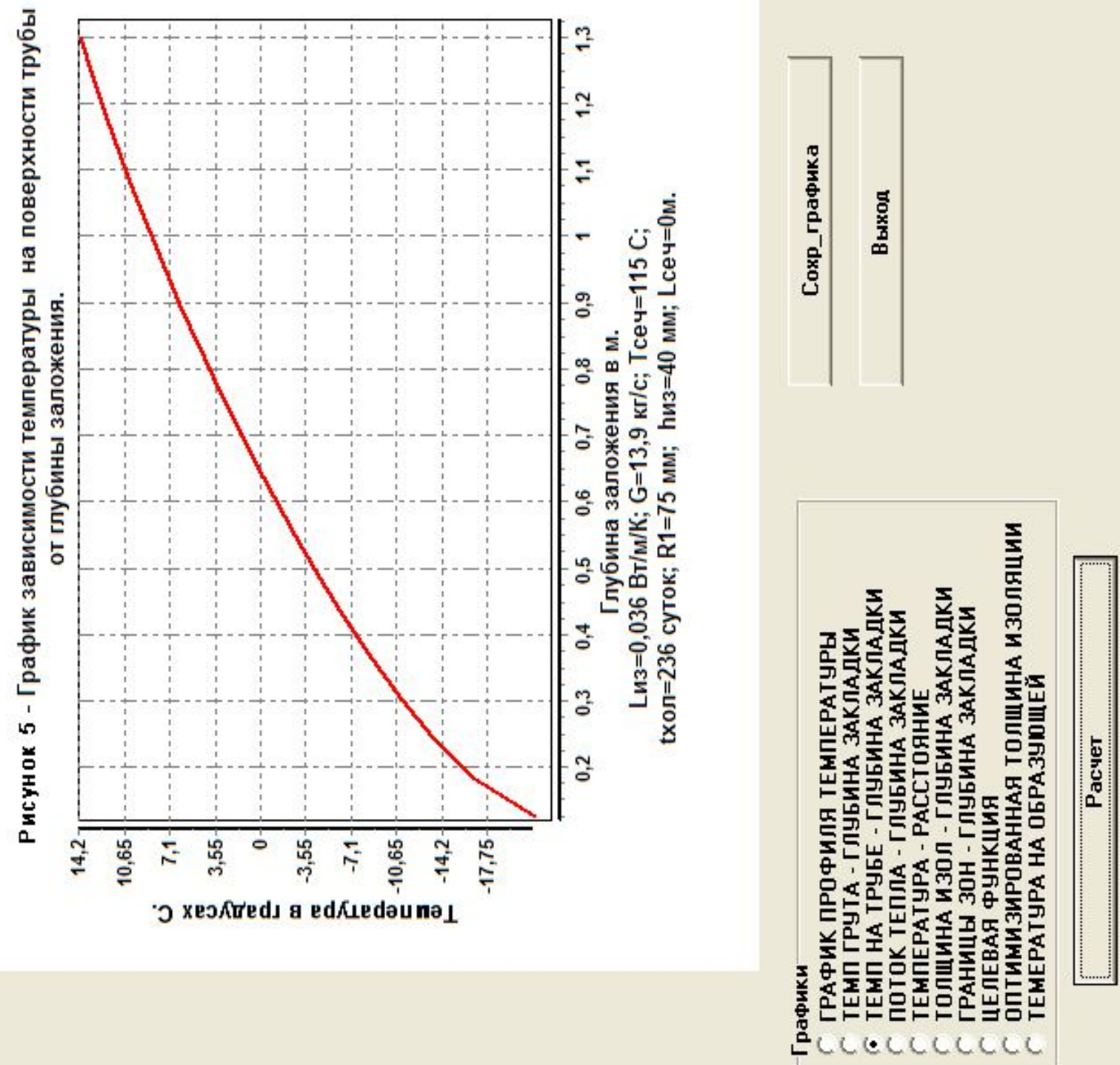

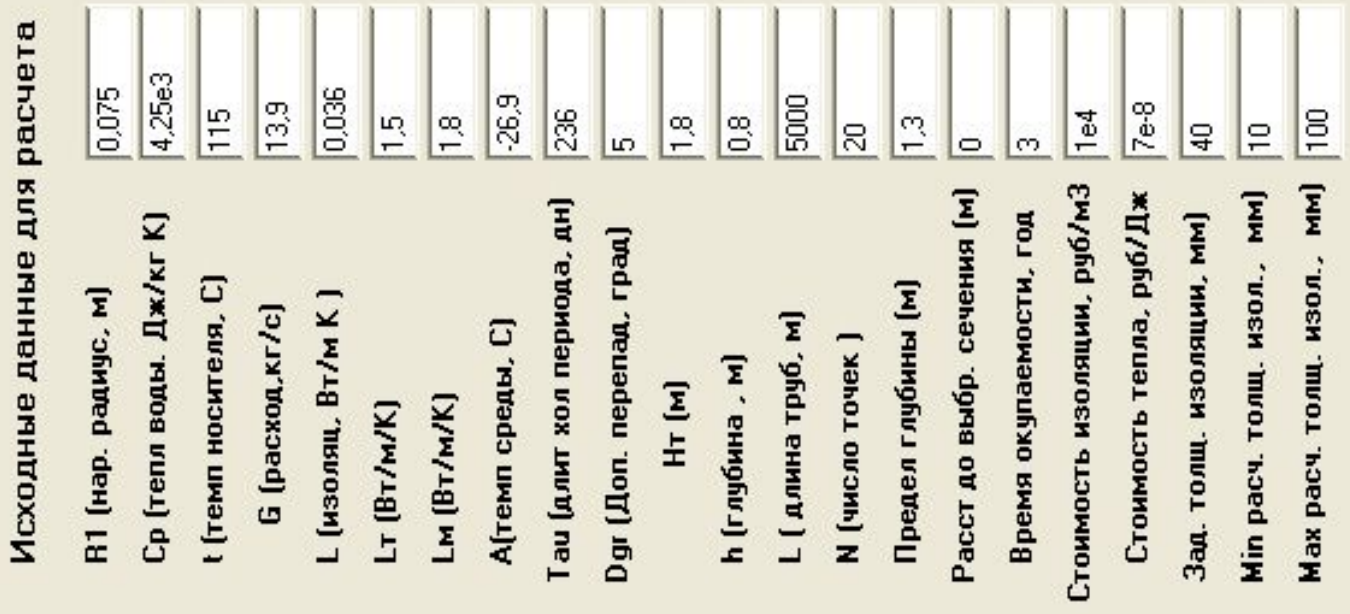



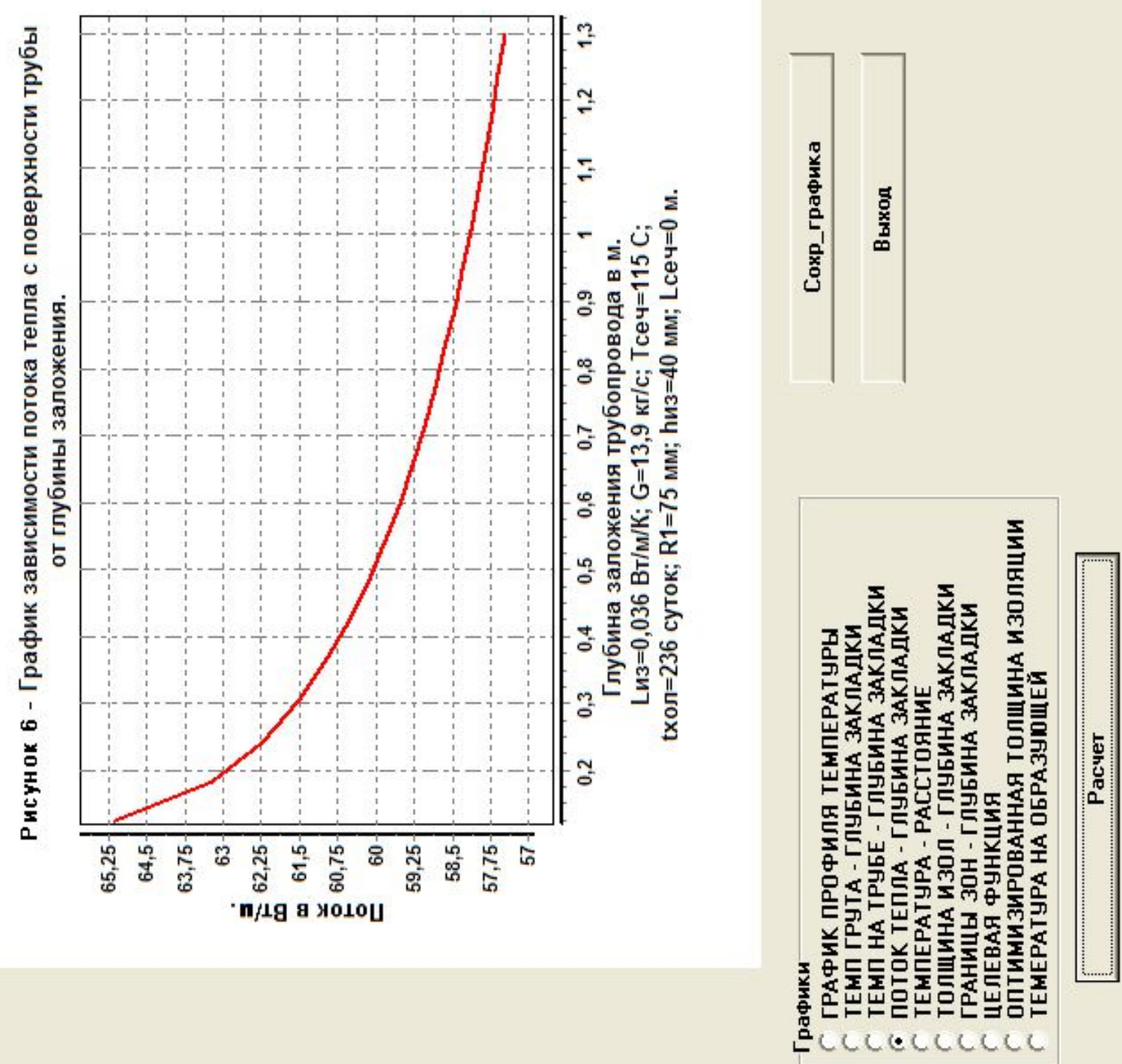

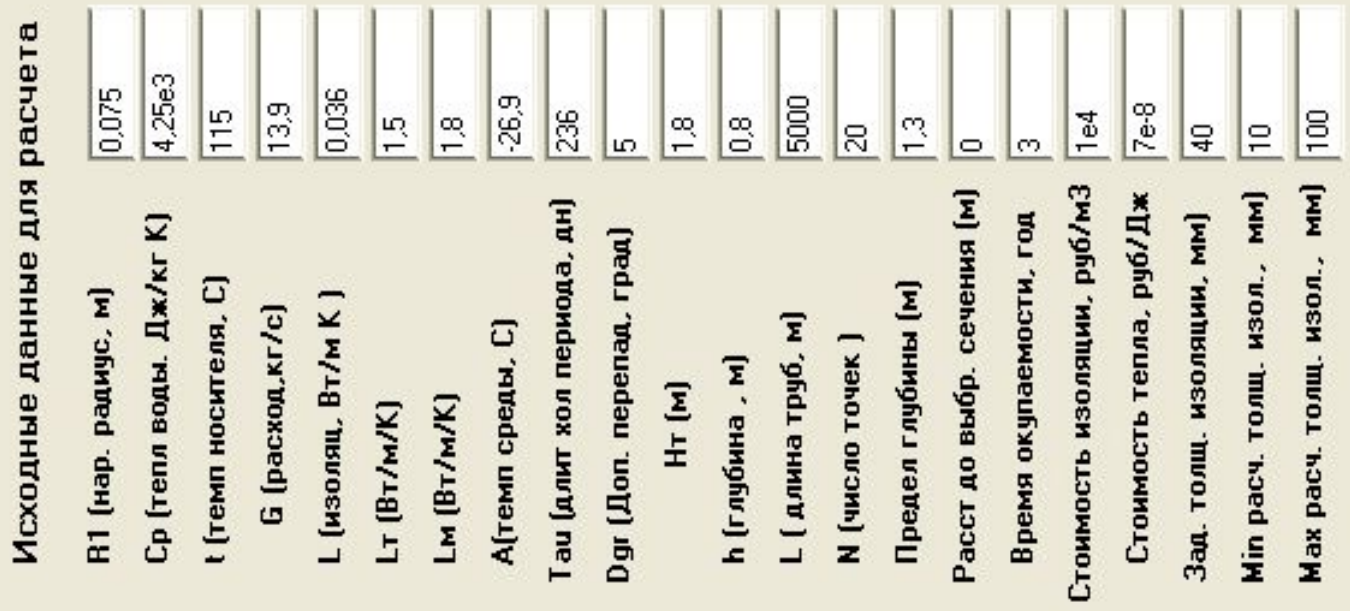



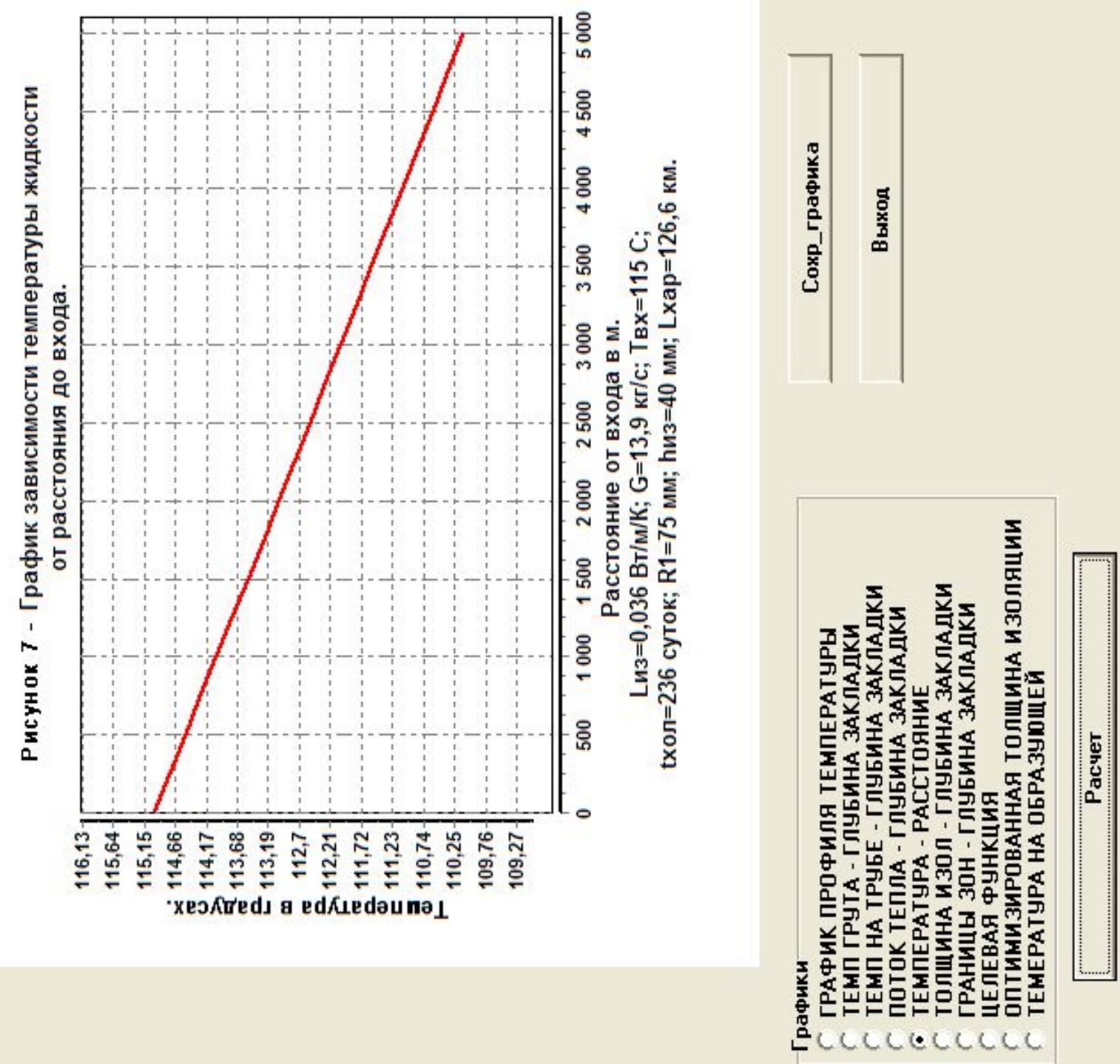

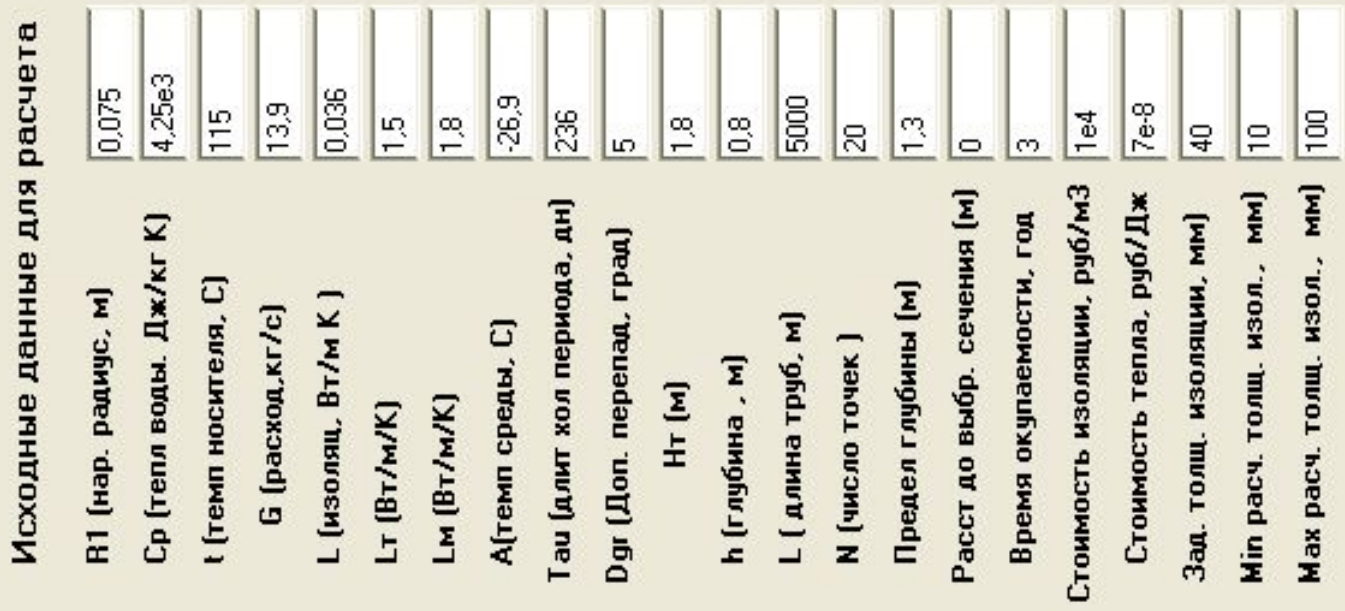


Связь с перепадом температур $\Delta \mathrm{t}_{0}$ и $\mathrm{t}^{\prime \prime}$ устанавливается из следующих соотношений. Так как

$$
\mathrm{t}_{\%}^{\prime \prime}(\mathrm{x})=\mathrm{t}_{\mathrm{rp}}+\left(\mathrm{t}^{\prime}-\mathrm{t}_{\text {гр }}\right) \exp \left(-\frac{\mathrm{L}}{1_{\text {хар }}}\right),
$$

то $\Delta \mathrm{t}_{0}=\left|\mathrm{t}^{\prime}-\mathrm{t}_{\text {\% }}^{\prime \prime}(\mathrm{x})\right|=\left(\mathrm{t}^{\prime}-\mathrm{t}_{\text {гр }}\right)\left(1-\exp \left(-\frac{\mathrm{L}}{1_{\text {хар }}}\right)\right)$,

где $\mathrm{t}^{\prime}$ - температура на входе, a $\mathrm{t}^{\prime \prime}$ - температура теплоносителя в пункте потребления. Отсюда найдем

$$
\exp \left(-\frac{\mathrm{L}}{1_{\text {хар }}}\right)=1-\frac{\Delta \mathrm{t}_{0}}{\left(\mathrm{t}^{\prime}-\mathrm{t}_{\mathrm{rp}}\right)} \text { или } \frac{\mathrm{L}}{1_{\text {хар }}}=-\ln \left(1-\frac{\Delta \mathrm{t}_{0}}{\mathrm{t}^{\prime}-\mathrm{t}_{\text {гр }}}\right) \text {, или } 1_{\text {хар }}=\frac{-\mathrm{L}}{\ln \left(1-\frac{\Delta \mathrm{t}_{0}}{\mathrm{t}^{\prime}-\mathrm{t}_{\mathrm{rp}}}\right)} .
$$

$\mathrm{Gc}_{\text {}} \mathrm{R}=\frac{-\mathrm{L}}{\ln \left(1-\frac{\Delta \mathrm{t}_{0}}{\mathrm{t}^{\prime}-\mathrm{t}_{\mathrm{rp}}}\right)}$

Зная общее сопротивление теплопередаче $\mathrm{R}$, а именно

$$
\begin{aligned}
& \mathrm{R}=\frac{-\mathrm{L}}{\mathrm{Gc}_{\text {ж }} \ln \left(1-\frac{\Delta \mathrm{t}_{0}}{\mathrm{t}^{\prime}-\mathrm{t}_{\mathrm{rp}}}\right)} \text { найдем из нелинейного уравнения } \\
& \mathrm{R}=\frac{\ln \frac{\mathrm{D}_{\mathrm{H}}+2 \delta_{\text {из }}}{\mathrm{D}_{\text {н }}}}{2 \pi \lambda_{\text {из }}}+\frac{\ln \left(\frac{2 \mathrm{~h}}{\mathrm{D}_{\mathrm{H}}+2 \delta_{\text {из }}}+\sqrt{\left(\frac{2 \mathrm{~h}}{\mathrm{D}_{\mathrm{H}}+2 \delta_{\text {из }}}\right)^{2}-1}\right)}{2 \pi \lambda_{\text {гр }}}
\end{aligned}
$$

величину $\delta_{\text {из }}$ с учетом того, что все остальные величины считаются фиксированными.

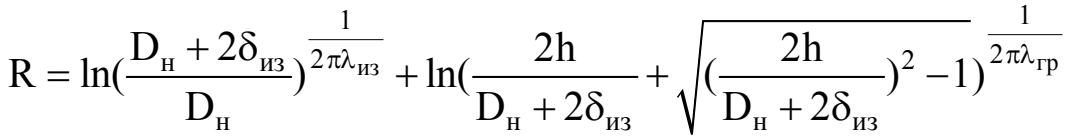

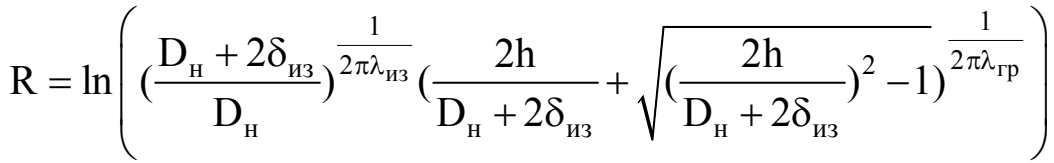

$$
\begin{aligned}
& \left(\frac{\mathrm{D}_{\mathrm{H}}+2 \delta_{\text {из }}}{\mathrm{D}_{\mathrm{H}}}\right)^{\frac{1}{2 \pi \lambda_{\text {из }}}}\left(\frac{2 \mathrm{~h}}{\mathrm{D}_{\text {н }}+2 \delta_{\text {из }}}+\sqrt{\left(\frac{2 \mathrm{~h}}{\mathrm{D}_{\mathrm{H}}+2 \delta_{\text {из }}}\right)^{2}-1}\right)^{\frac{1}{2 \pi \lambda_{\text {гр }}}}=\mathrm{e}^{\mathrm{R}}
\end{aligned}
$$

Корень данного уравнения $\delta_{\text {из }}$ был найден методом половинного деления.

Диалоговое окно (рис. 8) позволит определить зависимость толщины изоляции от глубины заложения трубопровода при заданном перепаде температур. 

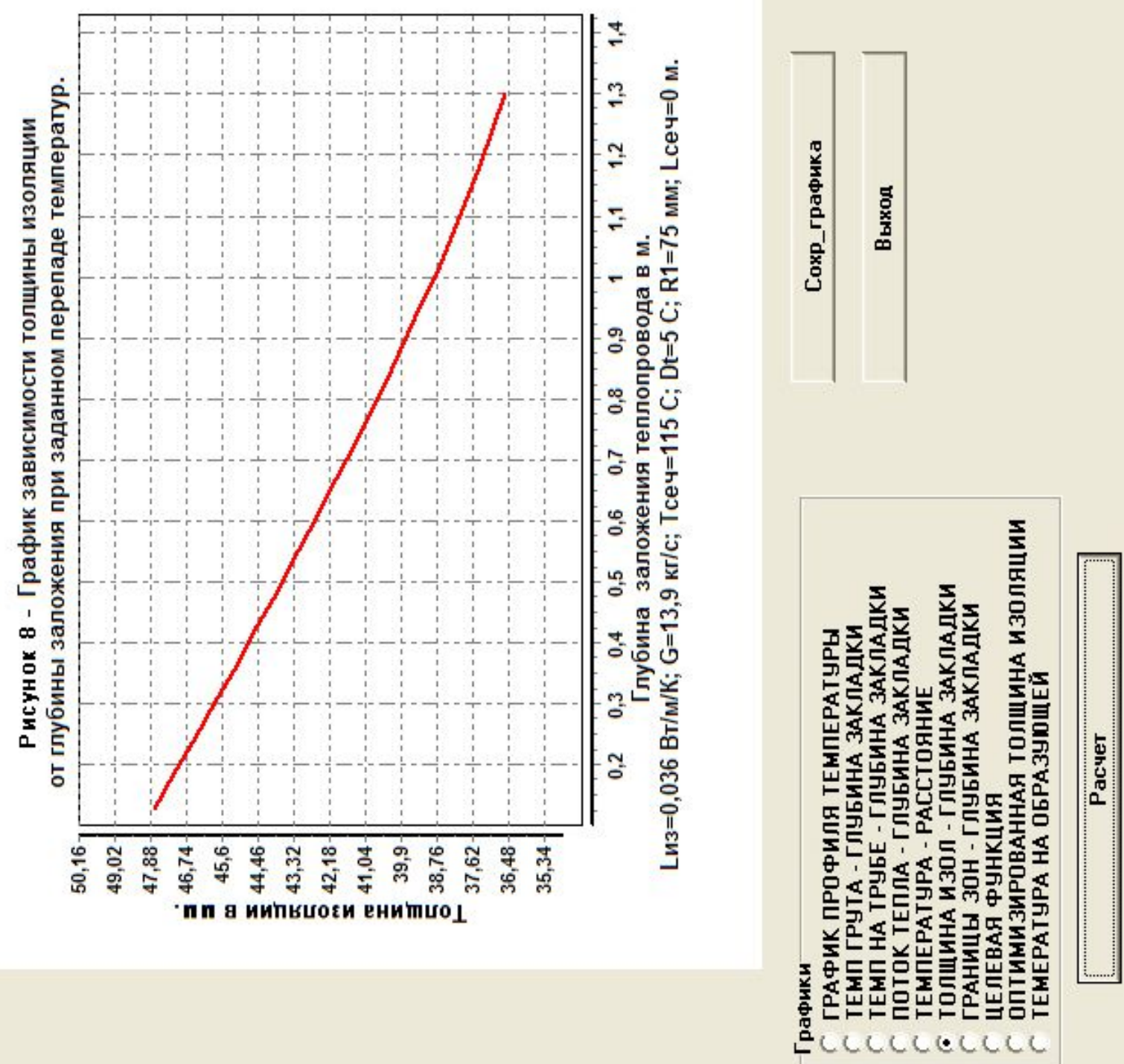

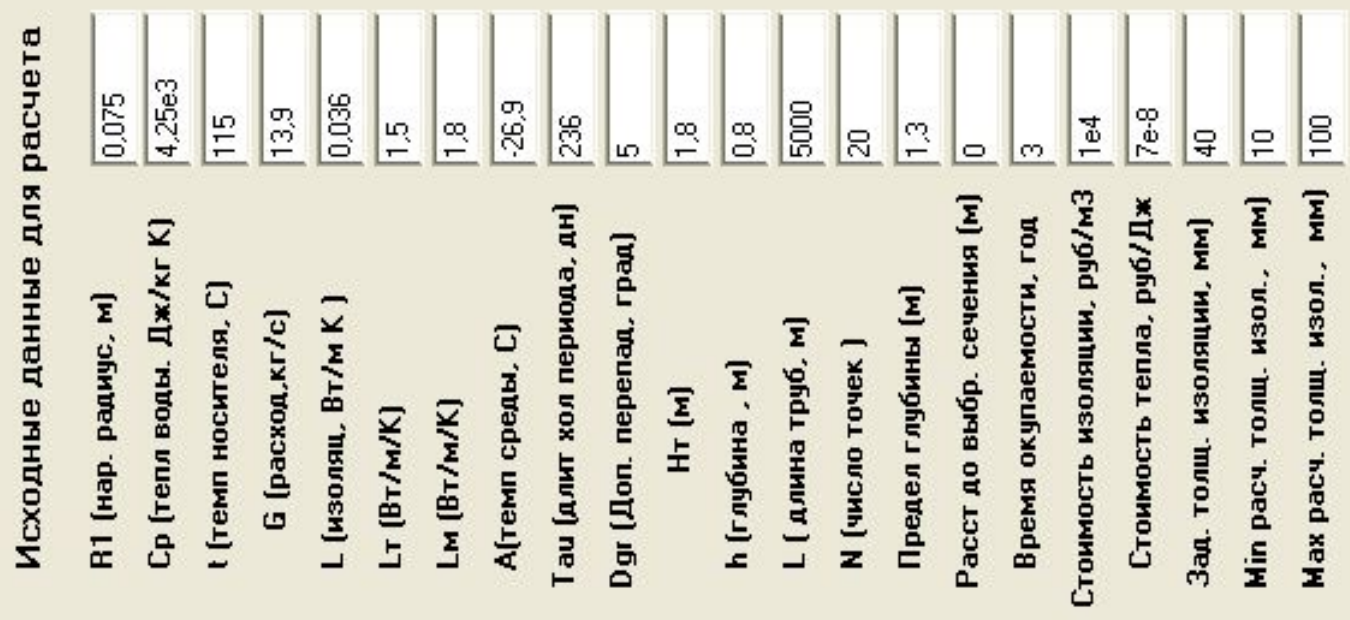


Расчет зависимости верхней и нижней зон оттаивания в зависимости от глу-

\section{бины заложения теплопровода}

Зная расчетные величины температур грунта и температуры на поверхности трубы из

$$
\frac{\mathrm{y}+\sqrt{\mathrm{h}^{2}-\mathrm{r}^{2}}}{\mathrm{y}-\sqrt{\mathrm{h}^{2}-\mathrm{r}^{2}}}= \pm \exp \left(-\frac{\mathrm{t}_{\text {гр }} \ln \left(\frac{2 \mathrm{~h}}{\mathrm{r}}\right)}{\frac{\lambda_{\mathrm{T}}}{\lambda_{\mathrm{M}}} \mathrm{t}_{\mathrm{T}}-\mathrm{t}_{\mathrm{гр}}}\right)
$$

определим, при разных знаках правой части, соответственно глубины верхней и нижней зон фронта оттаивания при разной глубине заложения. Из расчетов, проведенных выше, были определены равновесные температуры грунта и на поверхности трубы.

$$
\begin{aligned}
& \frac{y+\sqrt{h^{2}-r^{2}}}{y-\sqrt{h^{2}-r^{2}}}= \pm D \\
& y+\sqrt{h^{2}-r^{2}}= \pm D\left(y-\sqrt{h^{2}-r^{2}}\right) .
\end{aligned}
$$

Для варианта с плюсом

$$
\begin{aligned}
& \mathrm{y}+\sqrt{\mathrm{h}^{2}-\mathrm{r}^{2}}=\mathrm{D}\left(\mathrm{y}-\sqrt{\mathrm{h}^{2}-\mathrm{r}^{2}}\right) ; \\
& \mathrm{y}(1-\mathrm{D})=(-1-D) \sqrt{\mathrm{h}^{2}-\mathrm{r}^{2}}
\end{aligned}
$$

размер зоны определится частным следующего типа

$$
\mathrm{y}=\frac{(-1-\mathrm{D}) \sqrt{\mathrm{h}^{2}-\mathrm{r}^{2}}}{1-\mathrm{D}} .
$$

Для варианта с минусом

$$
\begin{gathered}
y+\sqrt{h^{2}-r^{2}}=-D\left(y-\sqrt{h^{2}-r^{2}}\right) ; \\
y(1+D)=(-1+D) \sqrt{h^{2}-r^{2}} ; \\
y=\frac{(-1+D) \sqrt{h^{2}-r^{2}}}{1+D}, \\
\text { где } \quad D=\exp \left(-\frac{t_{г p} \ln \left(\frac{2 h}{r}\right)}{\frac{\lambda_{T}}{\lambda_{M}} t_{T}-t_{г р}}\right) .
\end{gathered}
$$

Результаты расчета получим из диалогового окна (рис. 9). 

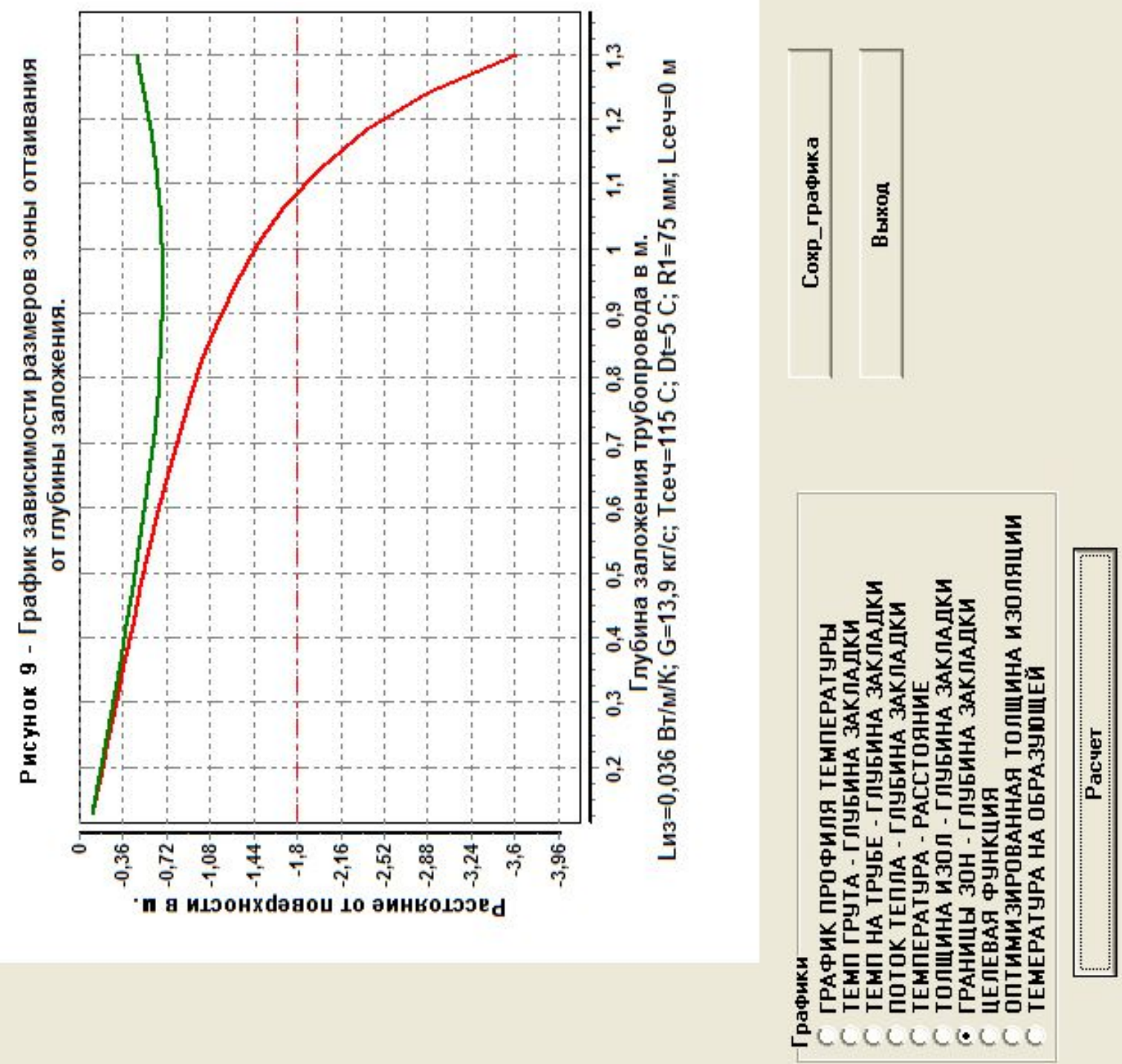

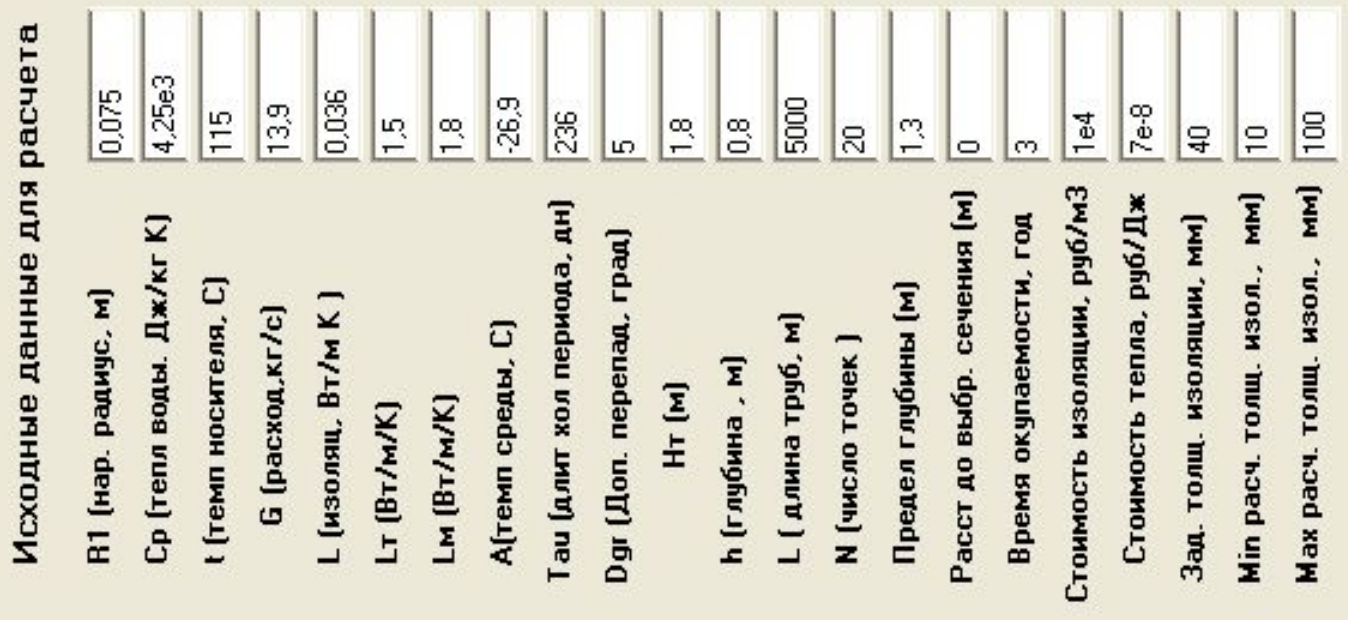


4. Технико-экономическая оценка при определении толщины изоляции теплопроводов

Тепловые сети в России характеризуются высокими потерями теплоты, поэтому одной из важнейших проблем современных систем централизованного теплоснабжения является снижение потерь теплоты трубопроводами тепловых сетей. В резерве энергосбережения доля мероприятий, связанных с тепловой изоляцией, составляет около 50 \%.

Повышение энергоэффективности тепловой изоляции систем централизованного теплоснабжения возможно только при использовании комплекса мероприятий, связанных с проектированием и выбором ее рациональных типов, толщины и свойств. В этой области накоплен обширный научный и практический потенциал. В то же время при всем многообразии возможных технических решений не существует единого подхода к оценке качества и эффективности тепловой изоляции систем централизованного теплоснабжения.

\section{1. Метод определения толщины изоляции, основанный на оптимизации} стоимостной целевой функции

При сооружении теплопроводов на севере Тюменской области возникает проблема определения оптимальной толщины теплоизоляции. В данной программе авторы предлагают метод определения толщины изоляции, основанный на оптимизации стоимостной целевой функции.

Если исходить из того, что известны стоимость изоляционного слоя $\mathrm{C}_{\text {из }}$ (в объемном выражении руб/куб метр) и $\mathrm{C}_{\mathrm{q}}$ стоимость теплоты (руб/Дж), то можно поставить задачу определения оптимальной толщины слоя изоляции при котором издержки на сооружение и эксплуатацию теплопровода будут минимальны, при этом будут выполнены условия СНиП.

Для некоторой длины теплопровода $1_{1}$ целевую функцию можно записать в виде следующих слагаемых:

$\Phi=\Phi_{0}+\Phi_{1}$

$\Phi_{0}=\Phi_{0}^{\prime}+\Phi_{\text {из }}-$ капитальные затраты на 1 м теплопровода, руб/м;

$\Phi_{\text {из }}$ - затраты на изоляцию;

$\Phi_{0}^{\prime}$ - прочие капитальные затраты;

$\Phi_{1}=\Phi_{1}^{\prime}+\Phi_{\mathrm{T}}-$ эксплуатационные затраты на 1 м теплопровода, руб/м;

$\Phi_{\mathrm{T}}-$ стоимость потерянной тепловой энергии;

$\Phi_{1}^{\prime}$ - прочие эксплуатационные расходы; 
$\left\{\begin{array}{l}\Phi_{\text {из }}=\frac{\pi}{4}\left[\left(\mathrm{~d}_{2}+2 \delta_{\text {из }}\right)^{2}-\mathrm{d}_{2}^{2}\right] \cdot Ц_{\text {из }} \\ \Phi_{\mathrm{T}}=\mathrm{q}_{1} \cdot \mathrm{T} \cdot Ц_{\mathrm{T}}=\frac{\mathrm{t}_{\text {ж }}-\mathrm{t}_{\mathrm{H}}}{\frac{1}{2 \pi \lambda_{\text {из }}} \cdot \ln \frac{\mathrm{d}_{2}+2 \delta_{\text {из }}}{\mathrm{d}_{2}}} \cdot \mathrm{T} \cdot Ц_{\mathrm{T}}\end{array}\right.$

$Ц_{\text {из }}-$ стоимость изоляции, руб/м ${ }^{3}$;

$Ц_{\text {т }}$ стоимость тепловой энергии, руб/Дж;

T - нормативный срок окупаемости, сек (год);

$\mathrm{q}_{1}=\frac{\mathrm{t}_{\text {ж}}-\mathrm{t}_{\mathrm{H}}}{\frac{1}{2 \pi \lambda_{\text {из }}} \cdot \ln \frac{\mathrm{d}_{2}+2 \delta_{\text {из }}}{\mathrm{d}_{2}}}-$ линейный тепловой поток, Вт/м.

Построение целевой функции приводится на рис. 10.

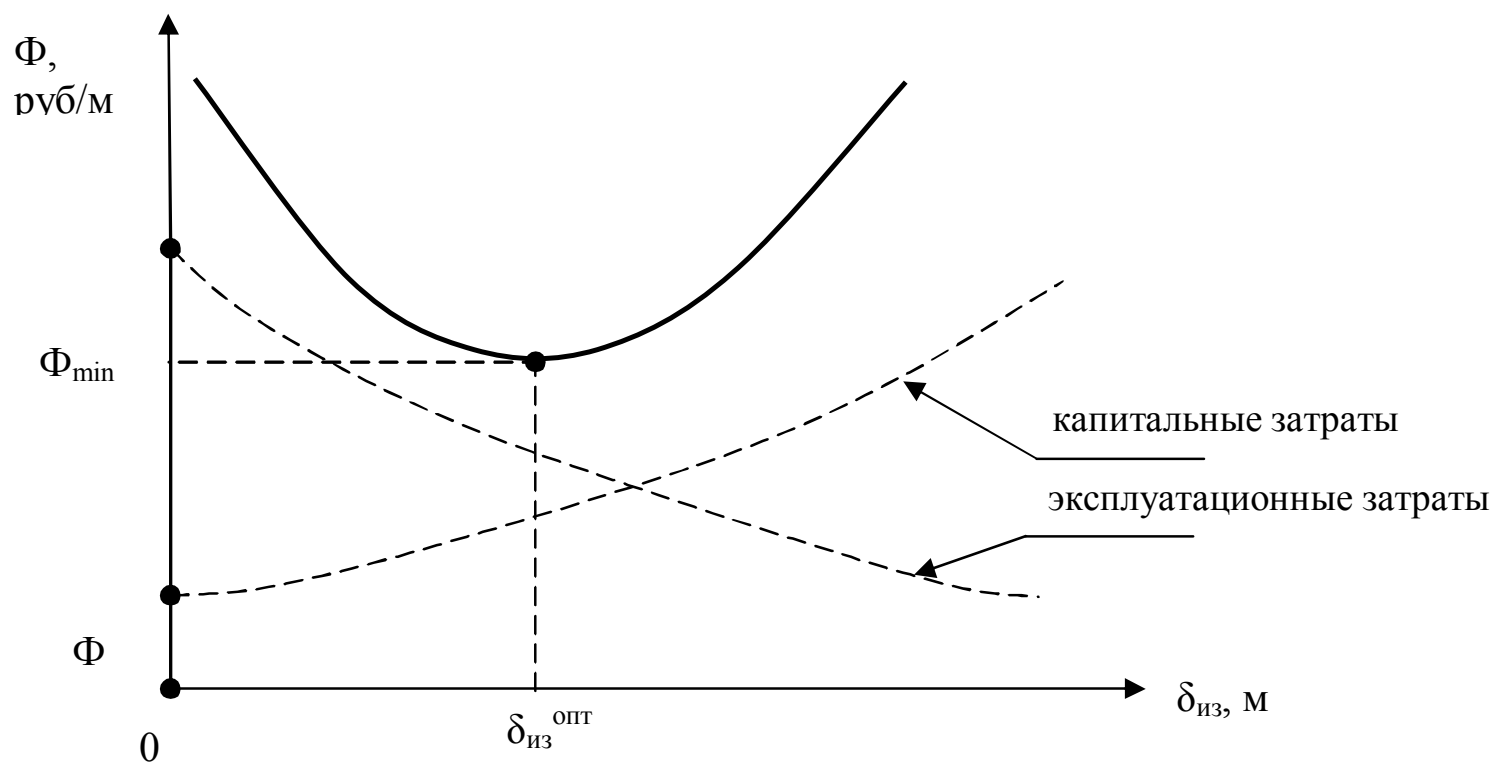

Рисунок 10 - Построение целевой функции

Поэтапная оптимизация целевой функции (алгоритм нахождения оптимальной толщины теплоизоляции)

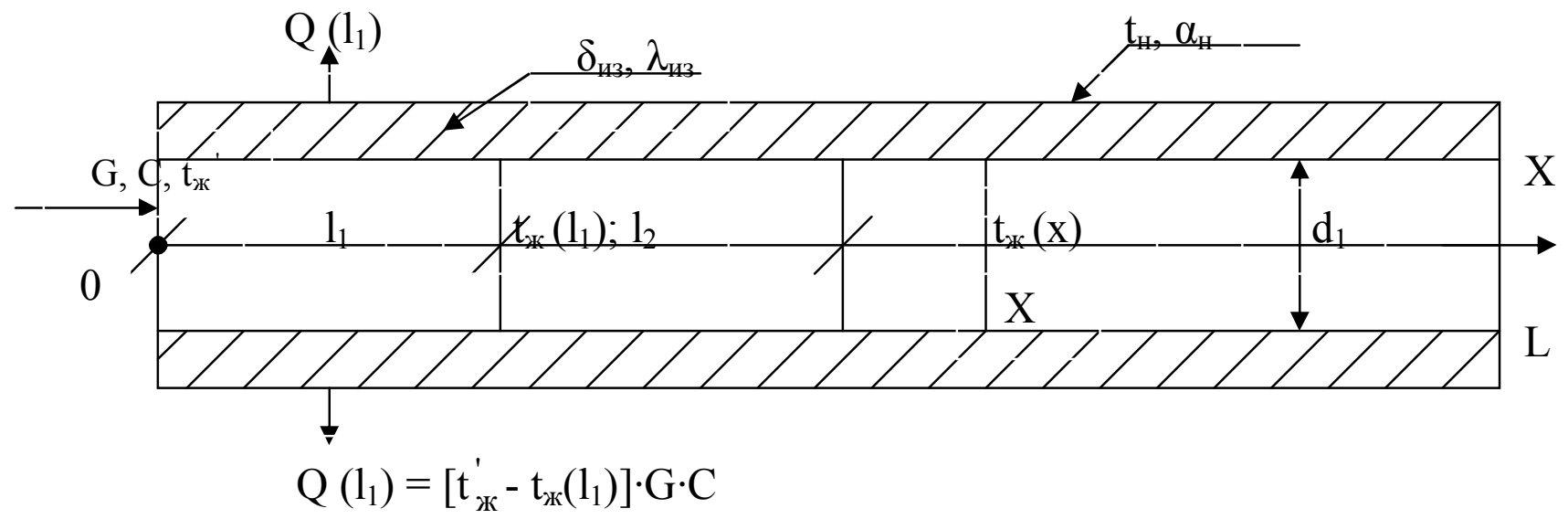

Рисунок 11 - Расчетная схема для разработки 
- Выбирается длина $1_{1}$ и определяются затраты на этом участке:

$$
\tilde{\Phi}\left(1_{1}\right)=\left(\Phi_{0}^{\prime}+\Phi_{1}^{\prime}\right) \cdot 1_{1}+\frac{\pi}{4}\left(4 \delta_{\text {из }} \cdot \mathrm{d}_{2}+4 \delta_{\text {из }}^{2}\right) \cdot Ц_{\text {из }} \cdot 1_{1}+\left(\mathrm{t}_{\text {ж }}^{\prime}-\overline{\mathrm{t}}_{\mathrm{H}}\right) \cdot\left[1-\exp \left(-\frac{1_{1}}{1_{\text {хар }}}\right)\right] \cdot \mathrm{G} \cdot \mathrm{C} \cdot Ц_{\mathrm{T}} \cdot \mathrm{T}
$$

- Условие минимума целевой функции $\widetilde{\Phi}\left(1_{1}\right)$

$$
\frac{\mathrm{d} \tilde{\Phi}}{\mathrm{d} \delta_{\text {из }}}=0 \rightarrow \delta_{\text {из }}=\delta_{\text {из }}^{\text {опт }}\left(1_{1}\right)
$$

- Находится температура теплоносителя при $\mathrm{x}=1_{1}$

$$
1_{\text {хар }}\left(1_{1}\right)=\frac{G \cdot C}{2 \pi \lambda_{\text {из }}} \ln \left[\frac{\mathrm{d}_{2}+2 \delta_{\text {из }}^{\text {опт }}\left(1_{1}\right)}{\mathrm{d}_{2}}\right] ; \quad \mathrm{t}_{\text {ж }}\left(\mathrm{l}_{1}\right)=\left(\mathrm{t}_{\text {ж }}^{\prime}-\overline{\mathrm{t}}_{\mathrm{H}}\right) \exp \left(-\frac{\mathrm{l}_{1}}{1_{\text {хар }}\left(\mathrm{l}_{1}\right)}\right)+\overline{\mathrm{t}}_{\mathrm{H}}
$$

- Переносим начало координат в точку $\mathrm{x}=1_{1}$, выбираем участок длины $1_{2}$ и повторяем предыдущие операции

- Выбор значений $1_{1}, 1_{2} \ldots$ определяется заданной точностью вычислений (в работе основные расчеты выполнены для $1_{1}=1_{2} \ldots=1 \mathrm{~m}$ )

Для выяснения «остроты» - минимума вычисляли по предложенной методике значения $\Phi_{1}(\mathrm{~L})$ для $1_{1}=\mathrm{L}$ при минимальном и максимальном значениях $\bar{\delta}_{\mathrm{i}, \text { из }}: \bar{\delta}_{\text {из }}^{\text {min }}=45,2$; $\Phi_{1}\left(\mathrm{~L}_{1}\right)=7,3$ млн. руб; $\bar{\delta}_{\text {из }}^{\max }=46,6 ; \Phi_{2}\left(\mathrm{~L}_{1}\right)=7,6$ млн. руб.

На рис. 12 приводится диалоговое окно зависимости целевой функции от толщины изоляции.

Анализ полученных значений целевой функции показывает, что минимум целевой функции является достаточно пологим, что обеспечивает свободу в выборе толщины изоляции. Проведенные исследования позволяют аналитически обосновать определение оптимальной толщины изоляции теплопроводов из диалогового окна (рис. 13).

В диалоговом окне (рис. 14) определяют зависимость температуры грунта на уровне боковой образующей от глубины заложения трубопроводов. 

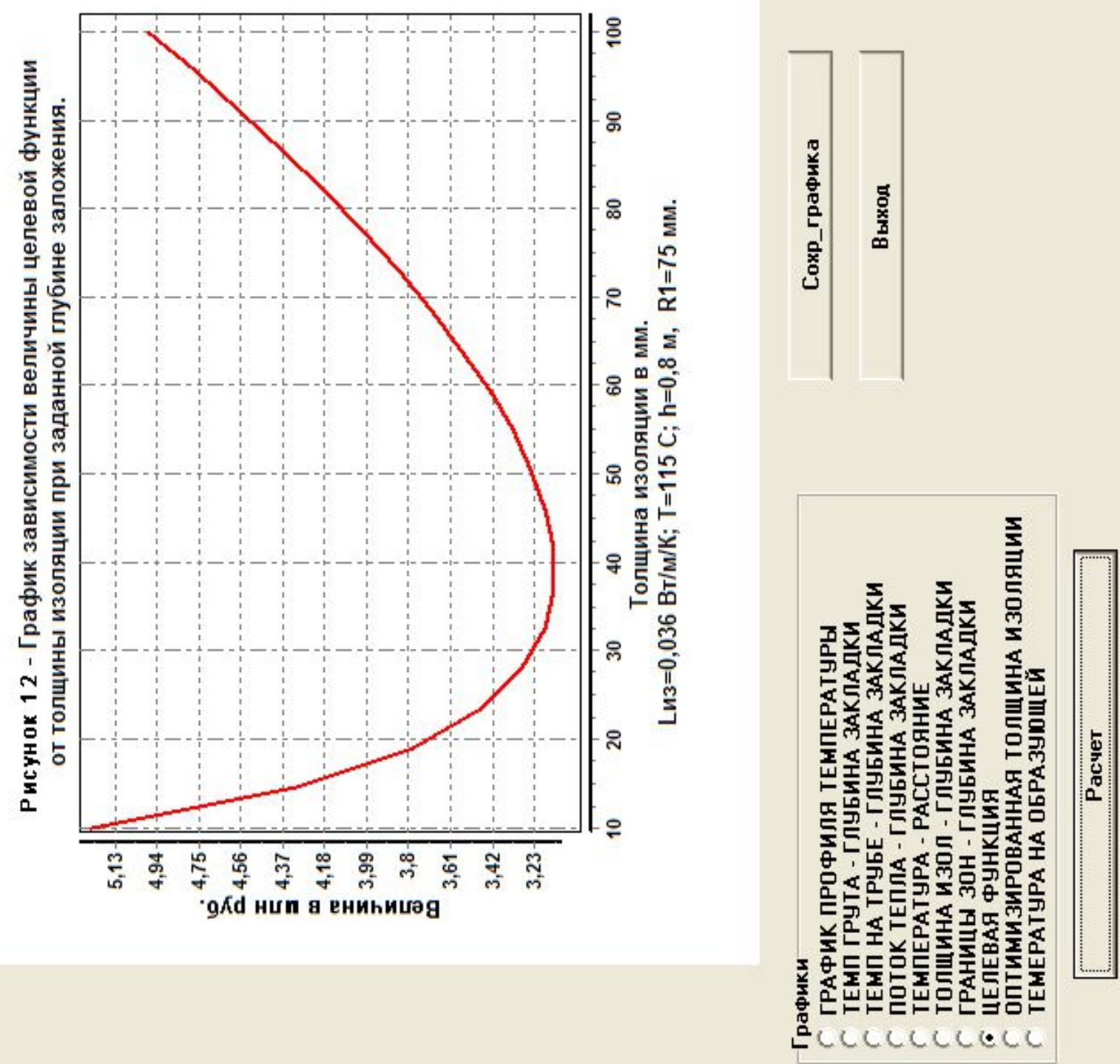

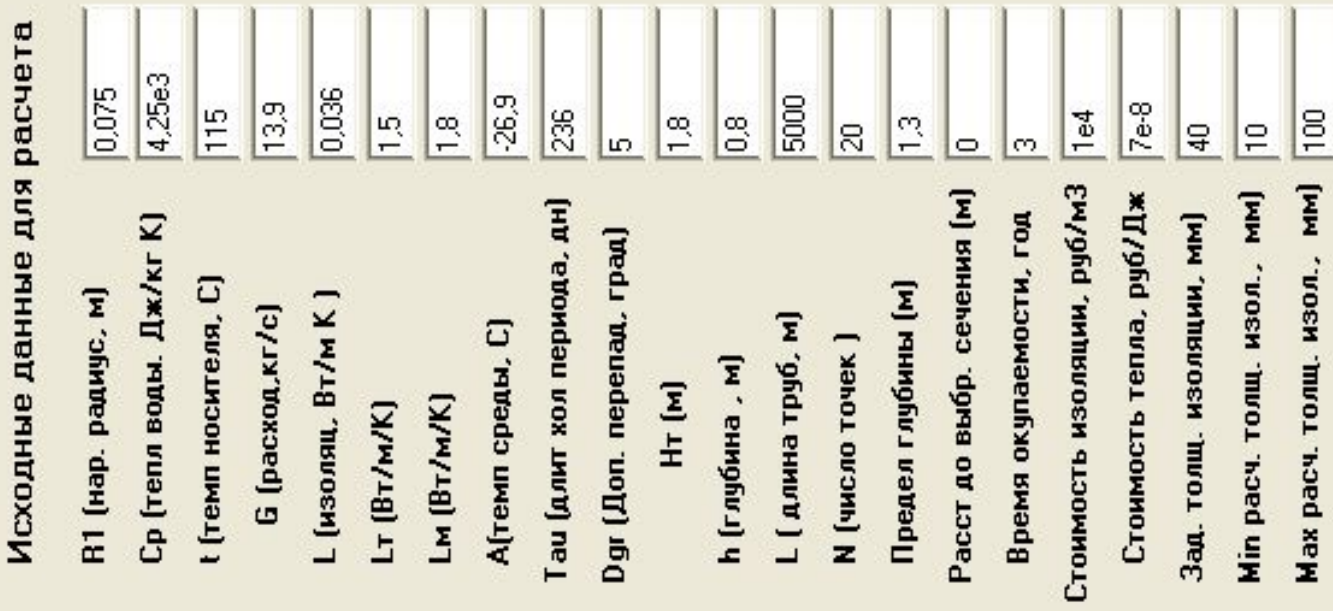



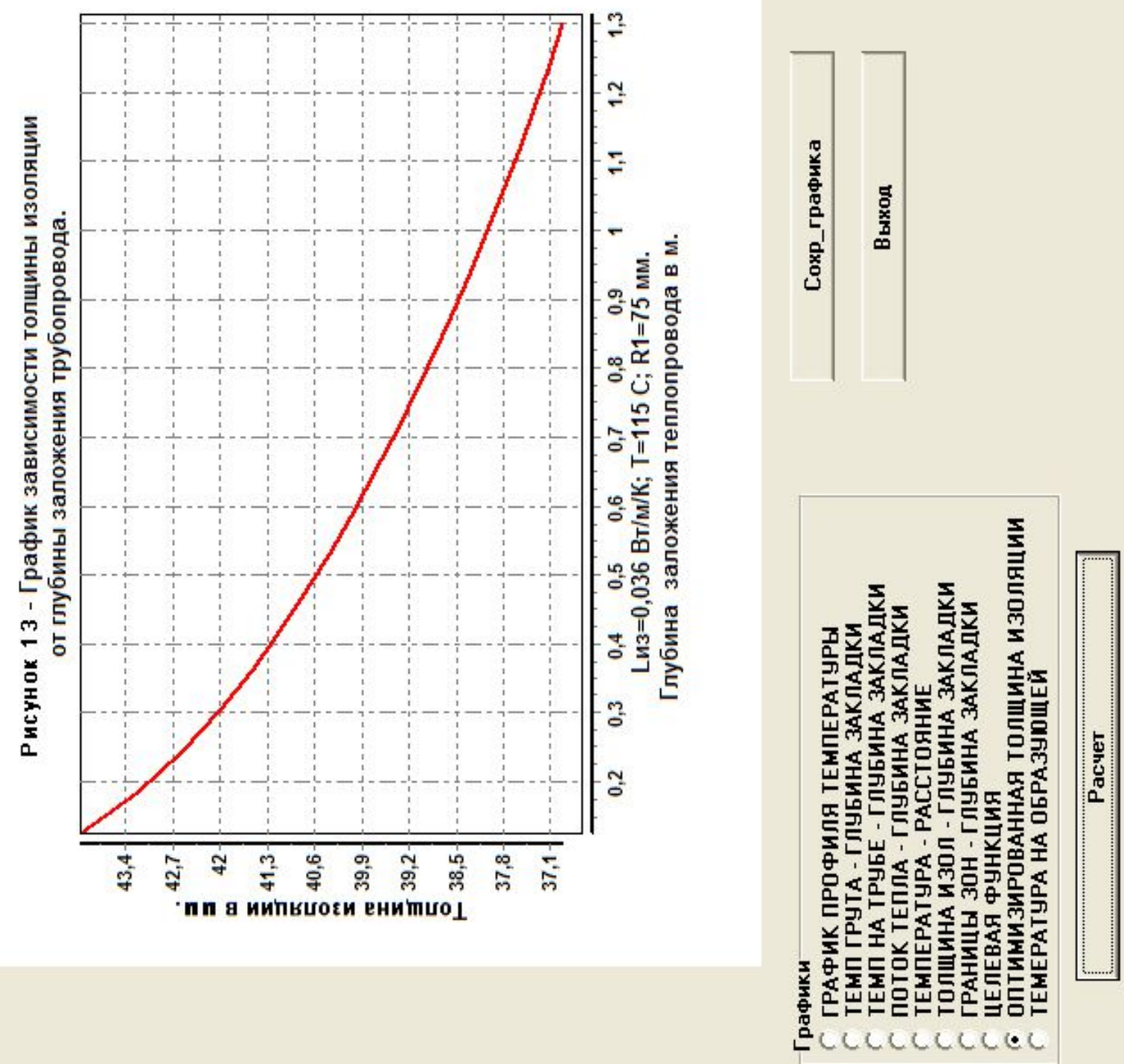

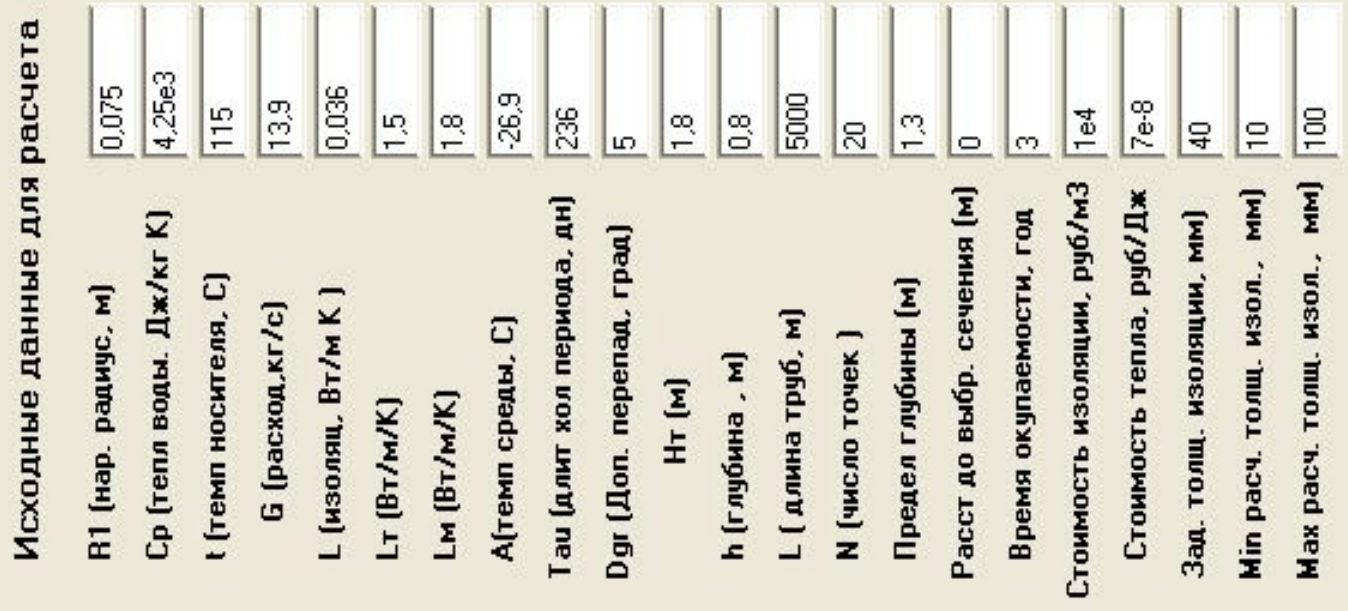



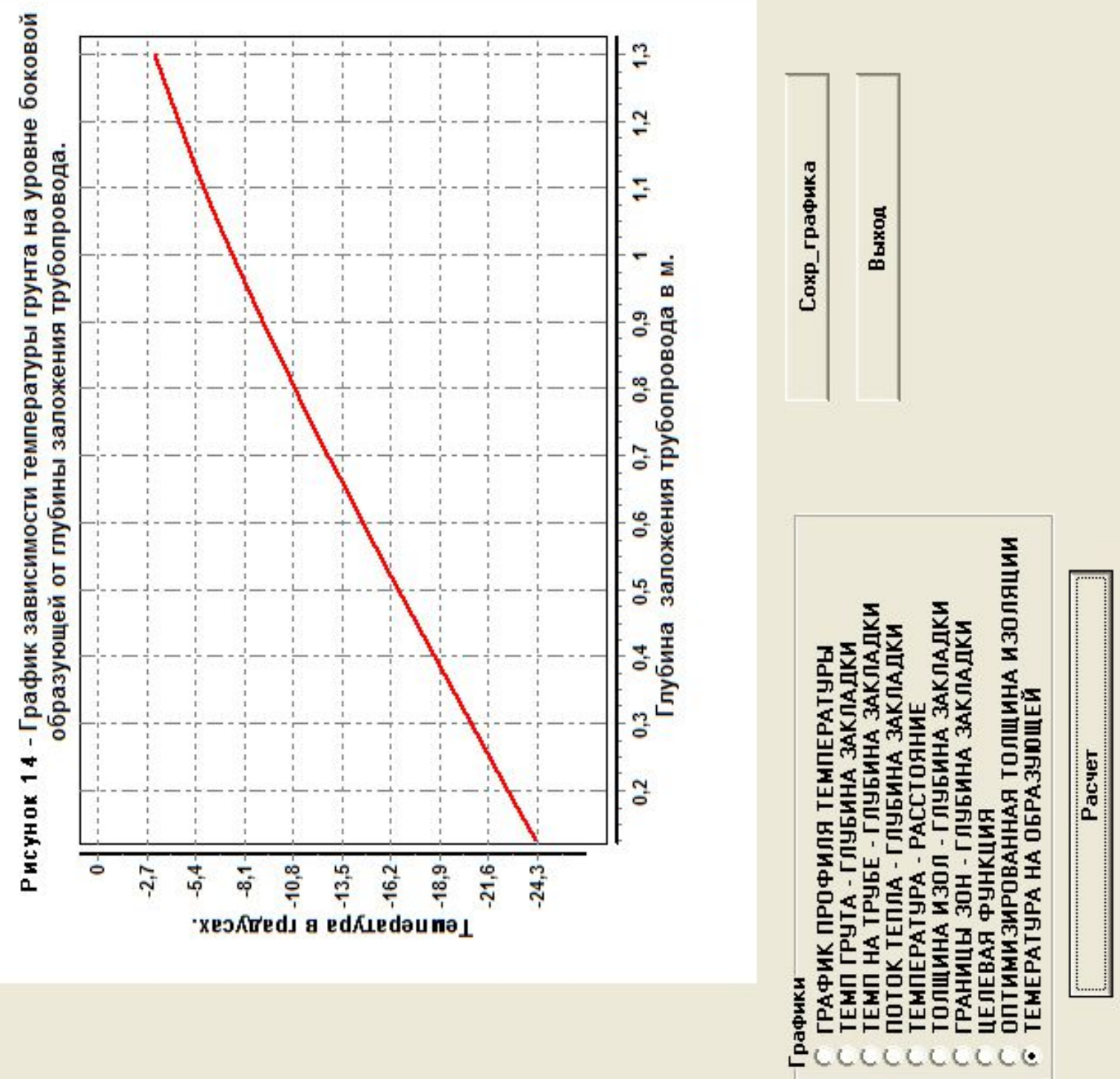

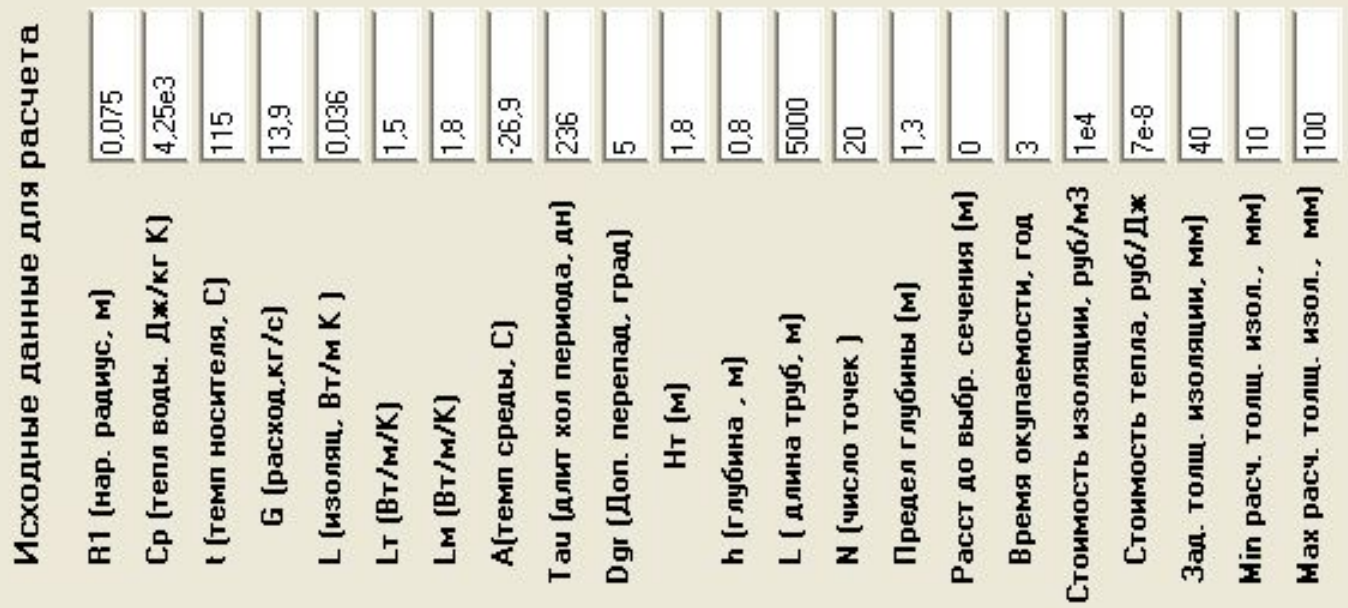




\section{5. Математическая модель температурного режима в ППУ-изоляции надзем-}

\section{ных теплопроводов}

Надземная прокладка выполняется по поверхности земли на низких опорах различных конструкций. Из анализа, проведенного авторами, этот способ является основным для незастроенной территории, а на промышленных площадках и в жилой зоне применяется там, где это допускается планировкой. Его применение уменьшает тепловое воздействие на мерзлый грунт, обеспечивает надежность работы, простоту устройства и удобство в эксплуатации теплопроводов.

Из анализа нормативных и расчетных потерь теплоты теплопроводами выявлены значительные расхождения. У проложенных надземным способом теплопроводов с ППУ изоляцией поверхность последней не нагревается даже настолько, чтобы растопить запорошенный их снег на трубопроводах.

Расчетная схема процесса теплового взаимодействия надземного теплопровода с окружающей средой, представлена на рис. 15. Распределение температур в слое изоляции трубопровода описывается дифференциальным уравнением в частных производных, которое в цилиндрических координатах имеет вид:

$$
\frac{\partial \mathrm{t}_{\mathrm{i}}}{\partial \tau}=\mathrm{a}_{\mathrm{i}}\left(\frac{\partial^{2} \mathrm{t}_{\mathrm{i}}}{\partial \mathrm{r}^{2}}+\frac{1}{\mathrm{r}} \frac{\partial \mathrm{t}_{\mathrm{i}}}{\partial \mathrm{r}}\right) .
$$

Для постановки и решения задачи были приняты следующие допущения: для определения среднегодовых теплопотерь температура теплоносителя согласно СНиП принимается средняя за год $-\mathrm{t}_{\mathrm{T}}=65{ }^{\circ} \mathrm{C}$; температура наружного воздуха согласно СНиП принимается средняя за год $\mathrm{t}_{\mathrm{o.c}}=-7,8{ }^{\circ} \mathrm{C}$ (г. Новый Уренгой). С учетом принятых допущений режим будет стационарный, и дифференциальное уравнение принимает вид:

$$
\frac{\mathrm{d}^{2} \mathrm{t}_{\mathrm{i}}}{\mathrm{dr}^{2}}+\frac{1}{\mathrm{r}} \frac{\mathrm{dt}_{\mathrm{i}}}{\mathrm{dr}}=0
$$

В этом случае температура является функцией одной координаты - r.

$$
\mathrm{r}_{\mathrm{i}-1} \leq \mathrm{r} \leq \mathrm{r}_{\mathrm{i}} ; \mathrm{i}=1,2, \ldots, \mathrm{N} \text {. }
$$

Тепловой поток на стенке трубы определяется из решения уравнения (38) при следующих граничных условиях:

- на внутренней поверхности теплопровода

$$
\lambda_{1}\left(\frac{\partial \mathrm{t}_{1}}{\partial \mathrm{r}}\right)_{\mathrm{B}}=-\alpha_{1}\left(\mathrm{t}_{\mathrm{T}}-\mathrm{t}_{1}\right), \mathrm{r}=\mathrm{r}_{0} ;
$$

- на стыках различных слоев изоляции

$$
\lambda_{\mathrm{i}} \frac{\partial \mathrm{t}_{\mathrm{i}}}{\partial \mathrm{r}_{\mathrm{i}}}=\lambda_{\mathrm{i}+1} \frac{\partial \mathrm{t}_{\mathrm{i}+1}}{\partial \mathrm{r}_{\mathrm{i}+1}}, \mathrm{r}=\mathrm{r}_{\mathrm{i}}(\mathrm{i}=1,2, \ldots, \mathrm{N}-1)
$$




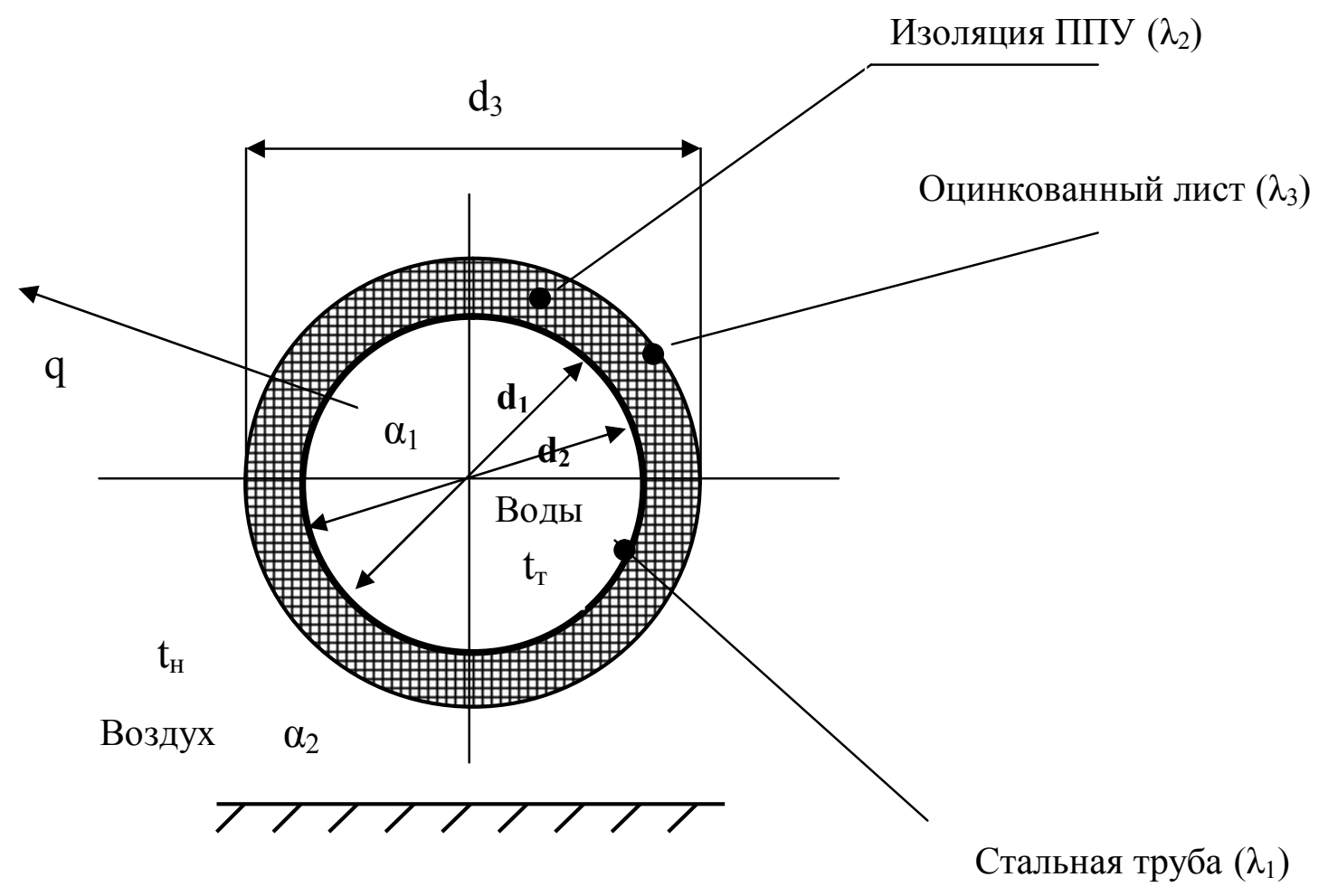

Рисунок 15 - Расчетная модель формирования температурного режима в ППУ-изоляции теплопровода и наружного воздуха

- на наружной поверхности стенки $\left(\mathrm{t}=\mathrm{t}_{\mathrm{H}}\right)$

$$
\lambda_{\mathrm{i}+1}\left(\frac{\partial \mathrm{t}_{\mathrm{i}+1}}{\partial \mathrm{r}_{\mathrm{i}+1}}\right)=-\alpha_{2}\left(\mathrm{t}_{\mathrm{T}}-\mathrm{t}_{\mathrm{H}}\right),
$$

где $\alpha_{1}-$ коэффициент теплоотдачи от теплоносителя к стенке теплопроводами, $\mathrm{BT} /\left(\mathrm{m}^{2} \cdot{ }^{\circ} \mathrm{C}\right) ; \mathrm{r}_{\mathrm{o}}$ - внутренний радиус теплопровода, м; $\alpha_{\mathrm{i}}$ - температуропроводность слоя теплоизоляции, $\mathrm{m}^{2} / \mathrm{c} ; \mathrm{t}$ - температура, ${ }^{\circ} \mathrm{C} ; \tau$ - время, $\mathrm{c} ; \mathrm{r}_{\mathrm{i}}$ - текущее значение радиуса трубы, м; $\alpha_{2}-$ коэффициент теплоотдачи от поверхности изоляционного слоя к наружному воздуху, Вт/( $\left.{ }^{2} \cdot{ }^{\circ} \mathrm{C}\right)$.

Согласно вышеизложенному на рис. 16-21 представлены следующие графики при надземной прокладке трубопровода:

• график зависимости толщины изоляции от расстояния;

- график зависимости температуры от расстояния;

- график зависимости целевой функции от толщины изоляции;

- график зависимости ( $\mathrm{L}$ - характер.) от расстояния;

- график зависимости потерь теплоты от расстояния;

- график суммарной стоимости $Ф(x)$ от расстояния. 


\section{Исходные данные для расчета}

\begin{tabular}{|c|c|}
\hline R1 [нар. радиус, M] & 0,0750 \\
\hline G (расход,кг/c) & 13,9 \\
\hline $\mathrm{L}$ [изоляц, Вт/m K ] & 0,036 \\
\hline Ср [тепл воды. Дж/кг К] & $4,25 \mathrm{e} 3$ \\
\hline ( [темп носителя, С] & 115 \\
\hline Токуп, год & 3 \\
\hline tн [темп среды, С] & -30 \\
\hline Сиз [ стоим изол, ру6/m3 ] & $1 \mathrm{e} 4$ \\
\hline Ст (стоим тепла, руб/Дж) ] & $7 e \cdot 8$ \\
\hline L [ длина тру6, м] & 5000 \\
\hline N [число точек ] & 20 \\
\hline Точность расчетов & $1 \mathrm{e}-3$ \\
\hline$L=$ const (pacct, $M$ ] & 2000 \\
\hline
\end{tabular}

Расчет

Графики

- Толщина-Расст

Temneparypa-Pacct

Цел. функ. для $\mathrm{L}=\mathrm{const}$

Lxap - pacct.

Поток - расст

Цел. функ. - расст.

Сохр_графика

Исходные данные для расчета

R1 [нар. радиус, M]

G $(p a c x o \alpha, k r / c)$

0,0750

$L$ [изоляц, Вт/M K ]

13,9

Ср (тепл воды. Дж/кг K)

0,036

Ср [тепл воды. Дж/кг

$4,25 \mathrm{e} 3$

( [темп носителя, C]

115

Tokyn, rog

3

tн [темп среды, C]

.30

Сиз [ стоим изол, ру6/m3]

Ст [стоим тепла, руб/Дж) ]

L [ длина тру6, м]

N [число точек ]

Точность расчетов

$L=$ const [pacct, $M$ )

$7 \mathrm{e}-$

$7 \mathrm{e}-8$

$1 \mathrm{e}-3$

2000

\section{Расчет}

\section{Графики}

Толщина-Расст.

- Temператypa - Расст

( Цел. функ. Аля $\mathrm{L}=\mathrm{const}$

Lxap-pacct.

Соток - расст

Цел. функ. - расст.

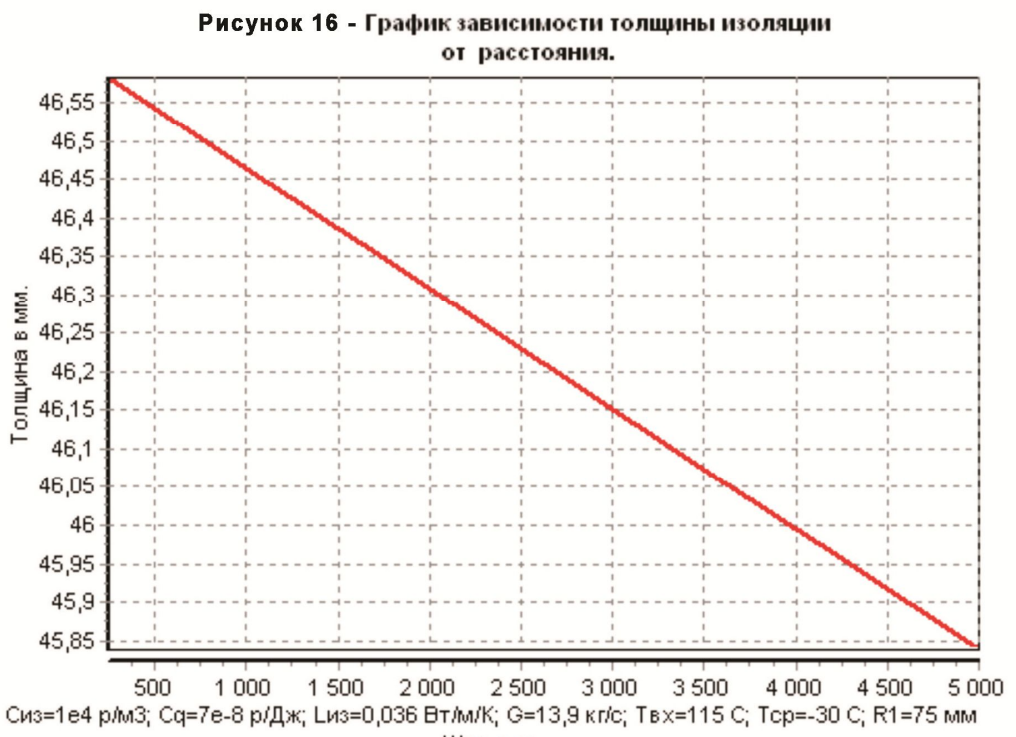

Шаг в M.

Каталог - Имя

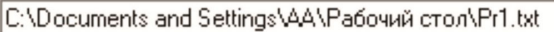

Coxp_pacчета_в_файл

Выxод

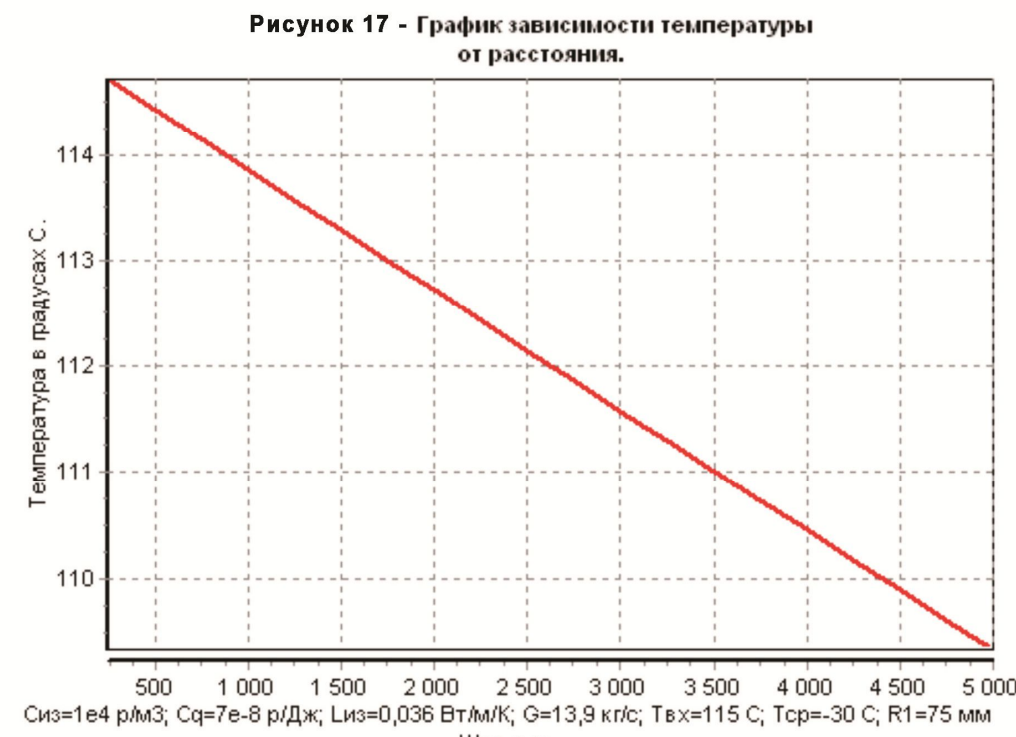
Шаr $\mathrm{B} \mathrm{M}$
Каталог - Имя

C: \Documents and Settings \АА

Сoxp_pacчета_в_файл 
Исходные данные для расчета

\begin{tabular}{|c|c|}
\hline R1 [нар. радиус, M] & 0,0750 \\
\hline G $[$ pacxoд,kr/c) & 13,9 \\
\hline L [изоляц, Вт/м K ] & 0,036 \\
\hline Ср [тепл воды. Дж/кг К] & $4,25 \mathrm{e} 3$ \\
\hline t (темп носителя, С] & 115 \\
\hline Tokyn, год & 3 \\
\hline tн [темп среды, С] & -30 \\
\hline Сиз [ стоим изол, ру6/m3 ] & $1 \mathrm{e} 4$ \\
\hline Ст [стоим тепла, ру6/Дж) ] & $7 \mathrm{e}-8$ \\
\hline L ( длина труб, м] & 5000 \\
\hline N [число точек] & 20 \\
\hline Точность расчетов & $1 e-3$ \\
\hline $\mathrm{L}=$ const $[$ pacct, $\mathrm{m}$ ] & 2000 \\
\hline
\end{tabular}

\begin{tabular}{c} 
Pacчet \\
Pan \\
\hline
\end{tabular}

Графики

Толщина-Расст

Temneparypa-Pacct

Сел. функ. для $\mathrm{L}=\mathrm{cons}$

Lxap - pacct.

- Поток - расст.

Цел. функ. - расст.

\section{Coxp_rрафика}

\section{Исходные данные для расчета}

R1 (нар. радиус, M)

$\mathrm{G}$ [расход,кr/c]

L (изоляц, Вт/M K ]

0,0750
139

Ср [тепл воды. Дж/кг К)

t [темп носителя, C]

Tokyn, год

0,036

$4,25 e 3$

15

tн [темп среды, C]

.30

Сиз [ стоим изол, py6/m3 ]

Ст [стоим тепла, руб/Дж ]

L ( длина тру6, м)

N [число точек ]

Точность расчетов

$L=$ const (pacct, $M$ )

$\sqrt{7 e-8}$
$\sqrt{5000}$
$\sqrt{20}$
$1 \mathrm{e}-3$
2000

Pacuet

\section{Графики}

Толщина-Расст.

(C) Temneparypa-Pacct

(5) Џел. функ. аля $\mathrm{L}=\mathrm{const}$

C Lxap- pacct.

C Поток- расст.

( Цел. функ. - расст.

Coxp_rрафика
Рисунок 20 - График зависиности Q(потерь) теплоты от расстояния

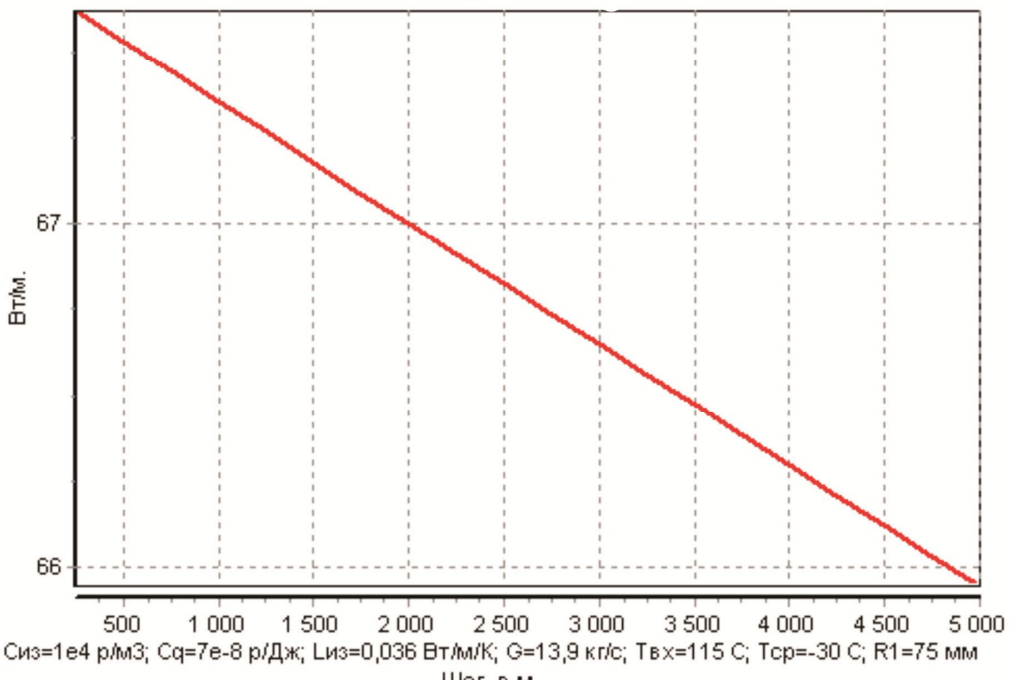

Шаr в m.
Каталог - Имя

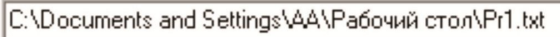

Сохр_расчета_в_файл

Выход

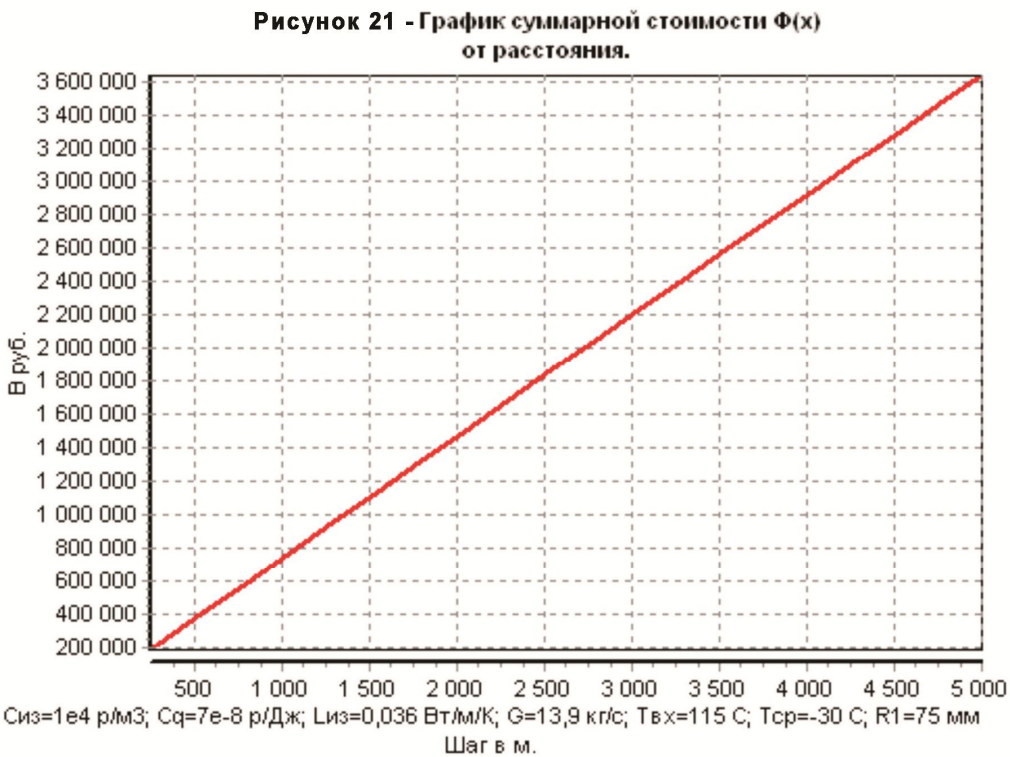

Каталог - Имя

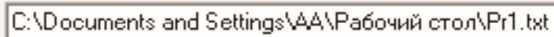

Coxp_pacчета_в_файл 


\section{Литература:}

1. Земенков Ю.Д., Моисеев Б.В., Илюхин К.Н., Налобин Н.В. Математическая модель температурного режима трубопроводов в вечномерзлых грунтах. «Известия вузов. Нефть и газ». - Тюмень, 2012, №4. - С. 96-99.

2. Илюхин К.Н., Налобин Н.В. Повышение энергоэффективности и снижение теплопотерь в системах теплоснабжения нефтегазовых объектов на севере Западной Сибири. - Спб.: ООО «Недра», 2007.- 114 с.:ил.

3. СНиП 41-03-2003. Тепловая изоляция оборудования и трубопроводов. - М.: Госстрой, 2004. - 29 с.

4. СНиП 41-02-2003. Тепловые сети. - М.: 2004. - 39 с.

5. Исследование тепловых процессов на объектах трубопроводного транспорта: Учебное пособие / Под общей редакцией Ю.Д. Земенкова. - Тюмень: Издательство «Вектор Бук», 2008. - 224 с.

\section{References:}

1. Zemenkov Yu.D., Moiseev B.V., Ilyukhin K.N., Nalobin N.V. Matematicheskaya model' temperaturnogo rezhima truboprovodov $\mathrm{v}$ vechnomerzlykh gruntakh (Mathematical model of temperature conditions of the pipelines in permafrost soils) // News of higher education institutions. Oil and gas. - 2012. - No. 4. - P. 96-99.

2. Ilyukhin K.N., Nalobin N.V. Povyshenie energoeffektivnosti i snizhenie teplopoter' v sistemakh teplosnabzheniya neftegazovykh ob'ektov na severe Zapadnoi Sibiri (Improvement of energy efficiency and reduction of heat losses in the heat supply systems of oil and gas facilities in the north of Western Siberia). - Saint Petersburg: Nedra LLC, 2007.

3. SNiP 41-03-2003 «Teplovaya isolyatsiya oborudovaniya i truboprovodov» («Heat insulation of equipment and pipelines»). - Moscow: Gosstroy, 2004. -29 p.

4. SNiP 41-02-2003 «Teplovye seti» («Heating systems»). - Moscow, 2004. - 39 p.

5. Examination of thermal processed at pipeline transport facilities: Training manual / Under general editorship of Yu.D. Zemenkov. - Tyumen: Vector Book Publishing House, 2008. $-224 \mathrm{p}$. 